This item was submitted to Loughborough's Research Repository by the author.

Items in Figshare are protected by copyright, with all rights reserved, unless otherwise indicated.

\title{
Tests for stationarity in series with endogenously determined structural
} change

PLEASE CITE THE PUBLISHED VERSION

LICENCE

CC BY-NC-ND 4.0

\section{REPOSITORY RECORD}

Harvey, David I., and Terence C. Mills. 2019. "Tests for Stationarity in Series with Endogenously Determined Structural Change". figshare. https://hdl.handle.net/2134/358. 
This item was submitted to Loughborough's Institutional Repository by the author and is made available under the following Creative Commons Licence conditions.

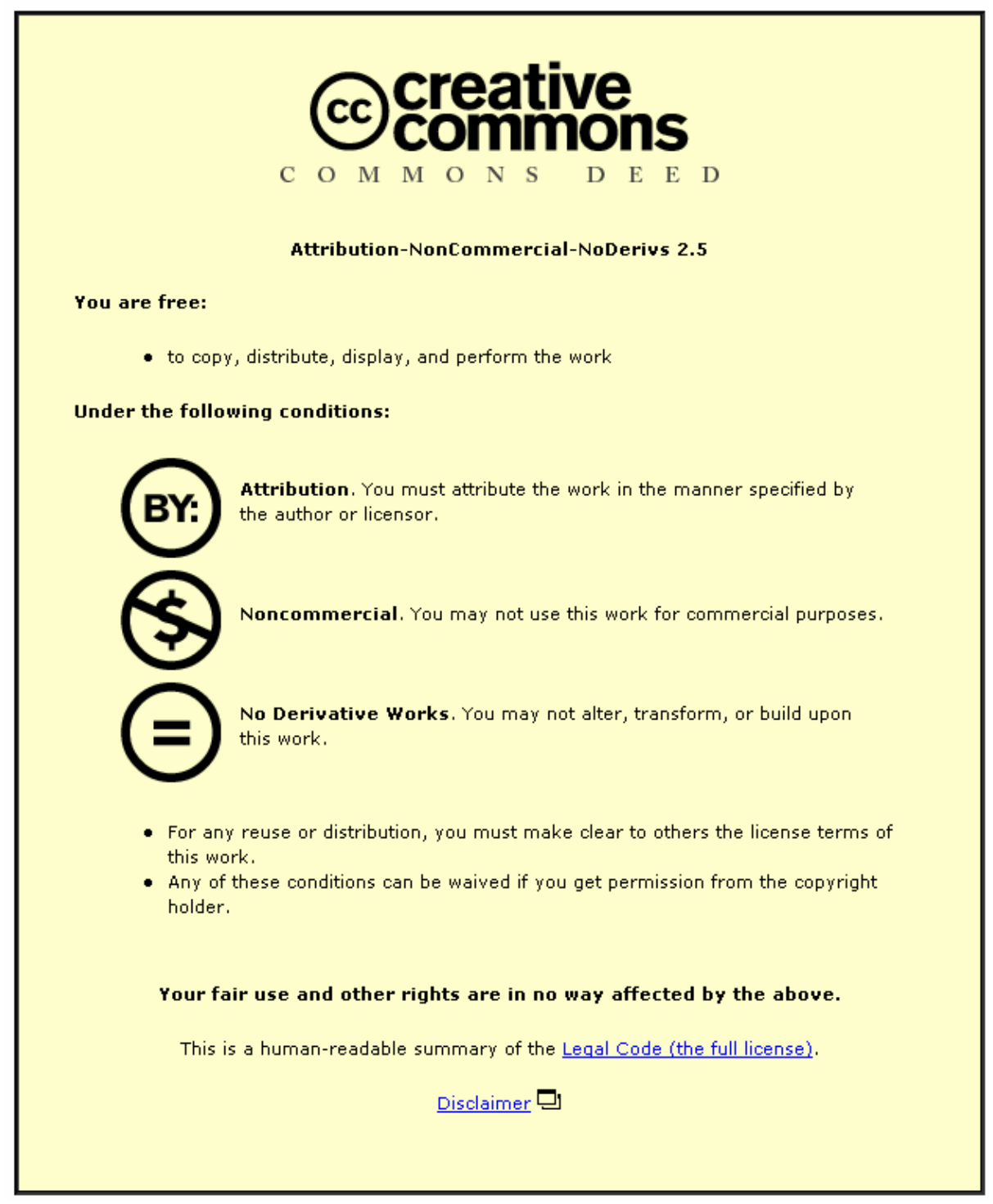

For the full text of this licence, please go to: http://creativecommons.org/licenses/by-nc-nd/2.5/ 


\title{
TESTS FOR STATIONARITY IN SERIES WITH ENDOGENOUSLY DETERMINED STRUCTURAL CHANGE
}

\author{
David I. Harvey ${ }^{\dagger}$ and Terence C. Mills \\ Loughborough University
}

\section{Summary}

We consider tests of the null hypothesis of stationarity against a unit root alternative, when the series is subject to structural change at an unknown point in time. Three extant tests are reviewed which allow for an endogenously determined instantaneous structural break, and a related fourth procedure is introduced. We further propose tests which permit the structural change to be gradual rather than instantaneous, allowing the null hypothesis to be stationarity about a smooth transition in linear trend. The size and power properties of the tests are investigated, and the tests are applied to four economic time series.

Keywords: Stationarity tests; Unit roots; Structural breaks; Smooth transitions. JEL classification: C12, C22.

\footnotetext{
${ }^{\dagger}$ Corresponding author: Department of Economics, Loughborough University, Loughborough, Leicestershire, LE11 3TU, UK. Tel.: +44 (0)1509 222712. Email: d.i.harvey@lboro.ac.uk
} 


\section{INTRODUCTION}

Hypothesis testing to determine whether economic time series are best modelled by (trend) stationary or unit root processes has been the subject of much recent research, and can be examined using two basic approaches. The first approach — "unit root testing" follows Dickey and Fuller (1979), and specifies the null hypothesis as a unit root process, testing against a stationary alternative. The second approach — "stationarity testing" reverses the null and alternative hypotheses, following tests first devised by Nyblom and Mäkeläinen (1983) and MacNeill (1978), and subsequently generalised to allow the series to follow a general ARMA or ARIMA process by Kwiatkowski et al. (1992)-hereafter KPSS - and Leybourne and McCabe (1994, 1999).

Modifications to both sets of procedures are required when the series is subject to a structural break. In the unit root testing framework, Perron $(1989,1993)$ first developed procedures to conduct testing when a break occurs at a known point in time, while several authors have considered more general techniques which assume that the break date is unknown and is to be determined endogenously (see, for example, Vogelsang and Perron, 1998, and Harvey et al., 2001). When the null hypothesis is that of stationarity with a structural break, Busetti and Harvey (2001) propose a KPSS-type test assuming the break point is known, while endogenous break date stationarity tests are developed by Lee (1999), Lee and Strazicich (2001) and Busetti and Harvey (2001, 2002).

In practice, the implicit assumption that any structural change occurs instantaneously may be unrealistic. An alternative approach in the unit root testing context is proposed by Leybourne et al. (1998), where the structural change is modelled by a smooth transition in linear trend. This procedure, which also allows the timing and speed of the transition to be determined endogenously, allows a series to gradually evolve from one trend regime to another, rather than undergo an instantaneous structural break.

In this paper we focus on stationarity tests in the presence of endogenously determined structural change. We review three extant instant break tests, and introduce a related fourth procedure, deriving the test's asymptotic distribution under the null. Further, we propose two tests of the null hypothesis that the series is stationary about a smooth transition in linear trend, paralleling the Leybourne et al. (1998) unit root testing work. The tests are then compared by way of Monte Carlo simulations, investigating size and power when instantaneous breaks and smooth transitions are present. The structure of the 
paper is as follows: Section 2 presents the instant break stationarity tests, while Section 3 proposes the smooth transition test procedures; the simulation experiments are examined in Section 4. In Section 5, the tests are applied to four economic time series: the $S \& P$ 500 stock market index, UK GDP, British industrial production, and the dollar-sterling exchange rate. The paper is concluded in Section 6 .

\section{STATIONARITY TESTS IN THE PRESENCE OF BREAKS}

Following Busetti and Harvey (2001), we consider four random walk models with different orders of deterministic components and structural breaks:

$$
\begin{array}{ll}
\text { Model } 1 & y_{t}=\mu_{t}+\delta_{\mu} w_{t}^{\prime}+\varepsilon_{t} \\
\text { Model 2 } & y_{t}=\mu_{t}+\beta t+\delta_{\mu} w_{t}^{\prime}+\delta_{\beta}\left(w_{t}^{\prime} t\right)+\varepsilon_{t} \\
\text { Model 2a } & y_{t}=\mu_{t}+\beta t+\delta_{\mu} w_{t}^{\prime}+\varepsilon_{t} \\
\text { Model 2b} & y_{t}=\mu_{t}+\beta t+\delta_{\beta} z_{t}^{\prime}+\varepsilon_{t}
\end{array}
$$

where $\mu_{t}=\mu_{t-1}+\eta_{t}$ with $\eta_{t} \sim \operatorname{NID}\left(0, \sigma_{\eta}^{2}\right), \varepsilon_{t} \sim \operatorname{IID}\left(0, \sigma^{2}\right), w_{t}^{\prime}=1\left(t>\tau^{\prime}\right)$ and $z_{t}^{\prime}=$ $1\left(t>\tau^{\prime}\right)\left(t-\tau^{\prime}\right)$, with $1($.$) being the indicator function and \tau^{\prime}$ the true break point. Model 1 has no trend component and a break in level, Model 2 contains a time trend and is subject to a simultaneous break in level and slope, while Models $2 a$ and $2 b$ also contain trends, but have breaks in level only, and slope only, respectively. The disturbance term in (1)-(4) can be generalised to allow for autocorrelation by permitting $\varepsilon_{t}$ to follow a more general stationary process, but for purposes of tractability in our analysis, we shall assume that this is unnecessary.

Busetti and Harvey (2001), and Lee and Strazicich (2001) for Models 1 and 2, propose

tests of the null hypothesis of stationarity $\left(H_{0}: \sigma_{\eta}^{2}=0\right)$ against a unit root alternative $\left(H_{1}: \sigma_{\eta}^{2}>0\right)$ when the true break date $\tau^{\prime}$ is unknown. If the true break date was known, the locally best invariant test would be a KPSS-type test constructed from the residuals of the appropriate regression based on (1)-(4):

$$
\xi_{i}\left(\lambda^{\prime}\right)=\frac{\sum_{t=1}^{T}\left(\sum_{s=1}^{t} e_{s}\right)^{2}}{T^{2} \hat{\sigma}^{2}} \quad(i=1,2,2 a, 2 b)
$$

where $e_{t}$ denotes the residuals from the appropriate regression among (1)-(4) above with $\mu_{t}$ replaced by a constant $\mu, \lambda^{\prime}=\tau^{\prime} / T$, and $\hat{\sigma}^{2}$ is the usual least squares estimator of $\sigma^{2}$. Now, treating the timing of the break as unknown, the Busetti-Harvey and Lee-Strazicich 
tests use an endogenously chosen break date, and their method follows the work of Zivot and Andrews (1992) in the unit root testing context. The test statistic (5) is calculated for all possible break dates $\tau$, and the break point which gives the most favourable result for the null of (trend) stationarity about a structural break is then selected, yielding the test statistic

$$
\tilde{\xi}_{i}=\inf _{\lambda \in \Lambda} \xi_{i}(\lambda) \quad(i=1,2,2 a, 2 b)
$$

where $\lambda=\tau / T$ and $\Lambda$ is a closed subset of the interval $(0,1)$. The break date is therefore implicitly estimated according to the criterion

$$
\hat{\tau}=\operatorname{argmin} \xi_{i}(\tau) \quad(i=1,2,2 a, 2 b)
$$

With regard to critical values for the tests, the authors take two different approaches. Busetti and Harvey (2001) derive asymptotic distributions under the null of stationarity for the test statistics (6) by making use of a "shift assumption" that the magnitudes of the breaks $\delta_{\mu}$ and $\delta_{\beta}$ in (1)-(4) decrease to zero with the sample size at rates faster than $T^{-1 / 2}$ and $T^{-3 / 2}$ respectively. (Clearly, this assumption encompasses the case where no break actually occurs.) These limiting distributions, along with the corresponding critical values, are given in Busetti and Harvey (2001), although the result for Model $2 a$ contains an error - the corrected distribution and critical values in this case are provided by Harvey and Mills (2002). These latter authors also provide finite sample critical values for all four models, and show that they differ little from their asymptotic counterparts.

Lee and Strazicich (2001), on the other hand, suppose that the true break date $\tau^{\prime}$ is selected by (7) asymptotically, and propose the use of critical values that correspond to tests with a break at a known point in time. Critical values - which depend on the value of $\lambda^{\prime}$-are thus drawn from the limiting distributions of $\xi_{i}\left(\lambda^{\prime}\right)$ in (5) and are reported for Models 1 and 2 in their paper, and for all four models in Busetti and Harvey (2001). In contrast to the above shift assumption approach, use of this procedure will not be valid in the case of no break, since the distribution of the test statistic is different when no break occurs.

In addition to the selection rule (7), other break date estimation procedures can be considered. Lee (1999) suggests use of the Schwarz Bayesian Criterion (SBC) in the wider context of potential multiple breaks, and uses the criterion to simultaneously determine the number of breaks as well as their timings. Busetti and Harvey (2002) propose esti- 
mating the relevant regression based on (1)-(4) for all possible break dates, and using the date which minimises the residual sum of squares; this procedure will clearly give identical estimates to Lee's SBC approach when only one break is considered.

In the parallel context of testing the null of a unit root in the presence of a break at an unknown time, selection procedures based on the significance of coefficients on the relevant break dummy variables have also been used (see, for example, Vogelsang and Perron, 1998). Indeed, Harvey et al. (2001), in analysing breaks in level, find that when a break occurs under the unit root null hypothesis, break date estimation based on dummy variable coefficient significance is superior to the Zivot and Andrews (1992) minimum test statistic approach, since the latter is subject to break date estimation problems when the magnitude of the break is large. The innovational outlier version of the unit root test also suffers from break date estimation problems when dummy variable coefficient significance is used, but Harvey et al. (2001) present a modification which overcomes this limitation. Moreover, this modification is found to outperform the use of SBC for innovational outlier tests when a break occurs under the null.

In the present context of testing a stationary null, break date selection based on the significance of break dummy variable coefficients translates to the following criteria, following estimation of the relevant regression based on (1)-(4) for all possible break dates:

$$
\begin{array}{ll}
\hat{\tau}=\operatorname{argmax}\left|t_{\hat{\delta}_{\mu}}(\tau)\right| & \text { for Models } 1,2 a \\
\hat{\tau}=\operatorname{argmax} F_{\hat{\delta}_{\mu}, \hat{\delta}_{\beta}}(\tau) & \text { for Model } 2 \\
\hat{\tau}=\operatorname{argmax}\left|t_{\hat{\delta}_{\beta}}(\tau)\right| & \text { for Model } 2 b
\end{array}
$$

where $t_{\hat{\delta}_{\mu}}(\tau)$ and $t_{\hat{\delta}_{\beta}}(\tau)$ are the $t$-ratios on $\delta_{\mu}$ and $\delta_{\beta}$ respectively, and $F_{\hat{\delta}_{\mu}, \hat{\delta}_{\beta}}(\tau)$ is the $F$-statistic for testing the joint significance of $\delta_{\mu}$ and $\delta_{\beta}$, all for a given break date $\tau$. Of course, unlike in the unit root testing framework, use of (8) will always give identical break date estimates to the minimum residual sum of squares and SBC criteria discussed above; this follows since no other regressors in (1)-(4) vary with the break date, so the break point associated with the most significant dummy variable coefficient(s) will necessarily coincide with the lowest sum of squared residuals. The stationarity test statistics which follow from this approach are simply obtained by calculating $\xi_{i}(\hat{\lambda})$ using $(5)$, where $\hat{\lambda}=\hat{\tau} / T$ with $\hat{\tau}$ obtained from (8).

As with the minimum stationarity test statistic procedure (6), there are two possible approaches to obtaining critical values for the test statistics $\xi_{i}(\hat{\lambda})$ based on $(8) /$ minimum 
residual sum of squares/SBC. One approach, which we develop here, is to maintain the shift assumption of Busetti and Harvey (2001) and derive limiting distributions of the test statistics under the null of stationarity. Using version (8) of these equivalent criteria to maintain consistency with the preferred approach in the unit root testing context, the null asymptotic distributions are (see Appendix for proof):

$$
\xi_{i}(\hat{\lambda}) \Rightarrow \int_{0}^{1}\left[B_{i}(r, \hat{\lambda})\right]^{2} d r \quad(i=1,2,2 a, 2 b)
$$

where the $B_{i}(r, \hat{\lambda})$ are as defined in Busetti and Harvey (2001) for $i=1,2,2 b$, and Harvey and Mills (2002) for $i=2 a$, except with $\lambda$ replaced by $\hat{\lambda}$, where

$$
\hat{\lambda}=\operatorname{argmax}_{\lambda \in \Lambda}\left|Z_{i}(\lambda)\right|
$$

with

$$
\begin{aligned}
Z_{1}(\lambda)= & \sqrt{\frac{\lambda}{1-\lambda}}\left[W(1)-\frac{1}{\lambda} W(\lambda)\right] \\
Z_{2}(\lambda)= & \frac{2}{\lambda^{3}(1-\lambda)^{5}}\left[\left(1-3 \lambda+3 \lambda^{2}\right) H_{1}^{2}+3\left(1-3 \lambda+4 \lambda^{2}\right)^{2} H_{1} H_{2}+3\left(1-2 \lambda+4 \lambda^{2}\right) H_{2}^{2}\right] \\
Z_{2 a}(\lambda)= & \sqrt{\frac{\lambda}{(1-\lambda)\left(1-3 \lambda+3 \lambda^{2}\right)}}\left\{W(1)-\frac{1}{\lambda} W(\lambda)-6(1-\lambda)\left[\int_{0}^{1} r d W(r)-\frac{1}{2} W(1)\right]\right\} \\
Z_{2 b}(\lambda)= & \sqrt{\frac{3}{\lambda^{3}(1-\lambda)^{3}}}\left\{\lambda^{2}\left[(\lambda-1) W(1)-\left[W(1)-\frac{1}{\lambda} W(\lambda)\right]\right]\right. \\
& \left.-(1-\lambda)^{2}(1+2 \lambda) \int_{0}^{\lambda} r d W(r)+\lambda^{2}(3-2 \lambda) \int_{\lambda}^{1} r d W(r)\right\}
\end{aligned}
$$

and

$$
\begin{aligned}
H_{1}= & 2 \lambda^{2}\left(1+\lambda+\lambda^{2}\right) W(1)-2 \lambda\left(1-2 \lambda+4 \lambda^{2}\right) W(\lambda)+3(1-\lambda)^{3} \int_{0}^{\lambda} r d W(r) \\
& -3 \lambda^{2}(1+\lambda) \int_{\lambda}^{1} r d W(r) \\
H_{2}= & -\lambda^{3}(1+\lambda) W(1)+\lambda\left(1-3 \lambda+4 \lambda^{2}\right) W(\lambda)-2(1-\lambda)^{3} \int_{0}^{\lambda} r d W(r)+2 \lambda^{3} \int_{\lambda}^{1} r d W(r)
\end{aligned}
$$

where $W(r)$ is a standard Wiener process. Asymptotic and finite sample critical values for these tests were obtained by Monte Carlo simulation and are given in Table I. The asymptotic critical values were generated by simulating the limiting distributions (9) using samples of $T=500$. For each replication, $Z_{i}(\lambda)$ was simulated for all possible break points considered, allowing calculation of $\hat{\lambda}=\operatorname{argmax}_{\lambda \in \Lambda}\left|Z_{i}(\lambda)\right|$ and simulation of $\xi_{i}(\hat{\lambda})$ for that replication. The space of values for $\lambda$ is restricted to a closed subset of $(0,1)$; in our simulations here and in Section 4, we restricted $\lambda$ to lie between the conventionally chosen points $(0.2,0.8)$. The finite sample critical values were obtained by repeated application 
of the test $\xi_{i}(\hat{\lambda})$, using (8) to estimate the break date, to a stationary generating process $\left(y_{t}=\varepsilon_{t}\right)$. With the exception of Model 1, the finite sample critical values are very close to those in the limit. Here and throughout the paper, simulations were programmed in GAUSS and conducted using 10000 replications.

The second approach, proposed by Lee (1999) and Busetti and Harvey (2002), is to use critical values associated with tests involving a break at a known point in time, making use of the fact that, when a break occurs, use of (8)/minimum residual sum of squares/SBC will choose the true break date $\tau^{\prime}$ superconsistently. As before, this procedure relies for its validity on a break actually being present, since the distribution is different when no break occurs.

\section{STATIONARITY TESTS IN THE PRESENCE OF SMOOTH TRANSITIONS}

The stationarity tests of the previous section all assume that when there is a break, the change occurs instantaneously. However, as argued by Leybourne et al. (1998) in the context of testing a unit root null hypothesis, this may be unrealistic in many economic applications. A more appealing approach would be to allow any structural change to occur gradually, with a smooth, rather than instantaneous, transition between two deterministic regimes. Leybourne et al. (1998) propose unit root tests where the alternative hypothesis is stationary about such a smooth transition in linear trend. These authors make use of the logisitic smooth transition function to model the change, following the work of Bacon and Watts (1971), Granger and Teräsvirta (1993) and Lin and Teräsvirta (1994).

Applying these concepts to the stationarity testing context, we can consider variants of the models (1)-(3) where the structural break terms are replaced by smooth transitions:

$$
\begin{array}{ll}
\text { Model } 1 & y_{t}=\mu_{t}+\delta_{\mu} S_{t}^{\prime}+\varepsilon_{t} \\
\text { Model 2 } & y_{t}=\mu_{t}+\beta t+\delta_{\mu} S_{t}^{\prime}+\delta_{\beta}\left(S_{t}^{\prime} t\right)+\varepsilon_{t} \\
\text { Model 2a } & y_{t}=\mu_{t}+\beta t+\delta_{\mu} S_{t}^{\prime}+\varepsilon_{t}
\end{array}
$$

where $\mu_{t}$ and $\varepsilon_{t}$ are as defined below (1)-(4) and $S_{t}^{\prime}$ is the logistic smooth transition function:

$$
S_{t}^{\prime}=\left[1+\exp \left\{-\gamma^{\prime}\left(t-\lambda^{\prime} T\right)\right\}\right]^{-1}
$$

The midpoint of the transition is given by $\lambda^{\prime} T$, and the transition speed is controlled by the parameter $\gamma^{\prime}$. At one extreme, $\gamma^{\prime}=0$ implies that no transition occurs at all, while in 
the limit $\gamma^{\prime} \rightarrow \infty$, the transition occurs instantaneously: thus no structural change and an instantaneous structural break are special cases of the above models. Smooth transition models do not apply well to the piecewise linear trend Model $2 b$, since this model relies on a single point at which the two regimes meet; a smooth transition variant of this type of process is better modelled by a transition in intercept and trend, i.e. Model 2.

Tests of the null hypothesis of stationarity, where the transition speed and timing are determined endogenously, can be conducted using a two stage procedure. In the first stage, the appropriate regression model based on (10)-(12) is estimated, with $\mu_{t}$ replaced by a constant $\mu$. This is done using a nonlinear least squares methodology, since the transition parameters $\gamma^{\prime}, \lambda^{\prime}$ are unknown. The residual sum of squares function can be minimised analytically over the regression parameters $\left(\mu, \beta, \delta_{\mu}, \delta_{\beta}\right)$ and numerically over the transition parameters $(\gamma, \lambda)$. In the second stage, a KPSS-type test statistic is computed:

$$
s_{i}=\frac{\sum_{t=1}^{T}\left(\sum_{s=1}^{t} e_{s}\right)^{2}}{T^{2} \hat{\sigma}^{2}} \quad(i=1,2,2 a)
$$

where $e_{t}$ denotes the residuals from the first stage regression. Conceptually, this method is most similar to the instant break stationarity tests where the break date is selected using (8), since in both cases the most likely timing of the structural change is explicitly determined as a first step, and then the stationarity tests are subsequently computed conditional on this choice. As with the instant break tests, the test statistics can be modified to allow for additional autocorrelation in the series, but here we shall assume that such augmentation is unnecessary.

Finite sample critical values for these tests are provided in Table II. These were obtained using Monte Carlo simulation, by repeatedly applying the tests of (14) to a stationary generating process $\left(y_{t}=\varepsilon_{t}\right)$. For each replication (here and in the Section 4 simulations), starting values for the nonlinear estimation were obtained from a grid-search procedure, with values for $\gamma$ and $\lambda$ given by $\gamma \in\{0.05,0.1,0.25,0.5,1\}$ and $\lambda \in\{0.1,0.2, \ldots, 0.9\}$ respectively. Numerical optimisation was performed using the Broyden, Fletcher, Goldfarb and Shanno algorithm in the OPTMUM subroutine for GAUSS; in cases where the algorithm produced estimates of $\gamma$ and $\tau$ which resulted in the transition function $S_{t}$ being constant for the whole sample, the Moore-Penrose generalised inverse was employed.

An alternative approach regarding critical values for the tests is to suppose that, for 
reasonably detectable transitions, the first stage optimisation will provide estimates of $\gamma$ and $\tau$ very close to their true values $\gamma^{\prime}$ and $\tau^{\prime}$, in which case critical values corresponding to tests with a smooth transition with known speed and midpoint can be employed. In contrast to the above approach, this procedure will not be valid when no transition in linear trend occurs. These critical values will depend on both $\gamma^{\prime}$ and $\tau^{\prime}$, and can be generated by simulation. By way of example, a grid of 5\%-level critical values for a sample size of $T=100$ are provided in Table III. These values were generated by repeated application of (14) to a generating model of stationarity about a smooth transition in linear trend, i.e. (10)-(12) with $\sigma_{\eta}^{2}=0$, treating the transition parameters as known in the first stage regressions.

\section{MONTE CARLO SIMULATIONS}

In order to examine the behaviour of the tests outlined in Sections 2 and 3, and compare their relative performance, we conducted a number of Monte Carlo experiments for a typical sample size of $T=100$. Let $\tilde{\xi}_{i}$ and $\xi_{i}(\hat{\lambda})$ denote tests using the stationarity test statistics with break date selection rules (7) and (8) respectively (as in Section 2), compared with critical values derived assuming the true break date is unknown, i.e. the finite sample critical values given in Harvey and Mills (2002) and Table I respectively. Similarly, let $s_{i}$ denote the smooth transition stationarity tests using critical values associated with a transition whose parameters are unknown, i.e. those in Table II. Then, let $\tilde{\xi}_{i}^{*}, \xi_{i}(\hat{\lambda})^{*}$ and $s_{i}^{*}$ denote tests using the same test statistics as above, but compared with critical values corresponding to tests where the timing (and speed for smooth transitions) of structural change is known, i.e. those of Table III for the smooth transition tests (in fact a slightly more detailed grid of values was used), and similarly simulated critical values for the instant break tests for a range of $\lambda^{\prime}$ values. The particular critical value used in a given application can be found by linear interpolation from these tables of critical values.

\subsection{Size and Power when an Instantaneous Break Occurs}

We first consider sizes of the tests when a break occurs under the null hypothesis. Table IV provides results of simulations for all six tests under each model specification with a range of break magnitudes and timings. The generating processes are given by (1)-(4) with $\sigma_{\eta}^{2}=0, \mu_{0}=\beta=0$ and $\varepsilon_{t} \sim \operatorname{NID}(0,1)$, with the exception that for Model $2, w_{t}^{\prime} t$ in 
(2) is replaced by $z_{t}^{\prime}$ so as to separate out the impacts of the level and trend components of the breaks. Note that since we do not consider a Model $2 b$ version of the smooth transition tests, the relevant entries in Panel $\mathrm{C}$ are for $s_{2}$ and $s_{2}^{*}$. Where they are comparable, our results and comments (here and for Table VI) concur with those of Busetti and Harvey (2002) and Lee and Strazicich (2001).

Examining first the instant break tests where unknown break point critical values are used, it can be seen that, although (by construction) both $\tilde{\xi}_{i}$ and $\xi_{i}(\hat{\lambda})$ have correct size when no break occurs, the tests suffer from size distortions when a break occurs under the null. The distortions are almost always those of oversize $\left(\xi_{1}(\hat{\lambda})\right.$ when $\lambda^{\prime}=0.5$ being the main exception) and vary according to the true break fractions $\lambda^{\prime}$ : for Models $1,2 b$ and 2 , distortions are more severe when the true break fraction is $\lambda^{\prime}=0.25$ or $\lambda^{\prime}=0.75$ than when a break occurs in the middle of the series, while this pattern is reversed for $\xi_{2 a}(\hat{\lambda})$. It appears, therefore, that the true distributions of the test statistics when breaks occur under the null are dependent on $\lambda^{\prime}$; this indicates that the shift assumption-under which the critical values used for these tests are derived - is violated for the break magnitudes considered in these simulations. Since breaks of this size are likely to arise in economic time series, the results question the validity of these tests in practical applications.

Comparing results for these two tests shows that, in almost all cases, the size distortions are more severe for $\tilde{\xi}_{i}$ than $\xi_{i}(\hat{\lambda})$, suggesting that use of (8) to estimate the break date is superior to use of (7). This feature is mirrored in the comparison of the tests using critical values associated with known break dates: whilst $\tilde{\xi}_{i}^{*}$ is dramatically undersized for all experiments, $\xi_{i}(\hat{\lambda})^{*}$ almost always has approximately the correct size. This last test therefore has substantial advantages in terms of size performance over the other instant break tests. The only case where size distortions occur is when there is no break under the null. As noted in Section 2, endogenous break tests using critical values corresponding to tests where the break date is known are not valid in such circumstances, and test undersize can be observed.

To further analyse the performance of the break date selection rules (7) and (8), Table $\mathrm{V}$ reports the percentage of times the true break date is chosen by the respective criteria for the same simulation experiments described above. The superior performance of (8) over (7) is dramatic for all models, with the correct break point chosen very frequently by (8) for even the smallest breaks, rising to $100 \%$ correct selection for larger break 
magnitudes. In contrast, the performance of (7) is always inferior, more so for Models 1 and $2 a$ than for Model $2 b$, whilst the true break date is especially poorly estimated for Model 2, even for large breaks in level and trend. Given that an incorrect choice of break date (even by one or two observations) has large implications for the resultant test size, it is these results that drive the superior size performance of $\xi_{i}(\hat{\lambda})^{*}$ over $\tilde{\xi}_{i}^{*}$ in Table IV.

Returning to Table IV, we can also evaluate the size behaviour of the smooth transition tests. The $s_{i}$ test is subject to size distortions which generally worsen with the magnitude of the break; these distortions persist for all cases except where no break occurs, in which case the test is correctly sized by construction. The pattern of oversize and undersize is very similar to that for the $\xi_{i}(\hat{\lambda})$ test, and the implication is that the critical values used for this test are inappropriate when breaks of interesting magnitude are present. The alternative test $s_{i}^{*}$, which uses critical values associated with tests where the transition midpoint and speed are known, has much better size performance, achieving approximate correct size in most cases. The test exhibits a little undersizing for small breaks and, as with the instant break tests of this type, is strongly undersized in the no break case. Considering the additional generality which this smooth transition test admits, the reliable size performance of $s_{i}^{*}$ in the presence of instant breaks is encouraging and signals potential value for applications.

It is also interesting to investigate the powers of the tests when an instant break occurs under the alternative hypothesis. Table VI provides results of such simulations for Model 1; these results are also representative of Models $2 a, 2 b$ and 2 with a similar overall picture obtained - a full set of results is available on request. The generating process is given by (1) with $\sigma_{\eta}^{2}=0.01,0.1,1, \mu_{0}=0$ and $\varepsilon_{t} \sim \operatorname{NID}(0,1)$. The same range of break magnitudes and timings are considered as with the null simulations, although the break sizes are scaled so as to represent 2.5, 5 and 10 times the standard deviation of the process each time. Moreover, the scaling is normalised so that if $\sigma_{\eta}^{2}=0$, the breaks would be identical to those used in the previous size experiments. Accordingly, the break magnitudes are specified as $\delta_{\mu}=\theta \sqrt{\left(\sigma_{\eta}^{2}+2\right) / 2}$ with $\theta=2.5,5,10$.

Consider first results for the four instant break tests. When $\sigma_{\eta}^{2}=1$ and the rejection frequencies are large, there is little difference in power among the tests; however, for smaller values of $\sigma_{\eta}^{2}$, clearer rankings can be observed. The tests that generally have highest power are those using unknown break date critical values, with $\tilde{\xi}_{1}$ outperforming 
$\xi_{1}(\hat{\lambda})$ when the break magnitude is moderate or zero, although this advantage disappears for larger break sizes. The problem with these procedures is that the high power comes at a price of size distortions when breaks occur under the null. Of the two tests using known break date critical values, $\xi_{1}(\hat{\lambda})^{*}$ has uniformly more power than $\tilde{\xi}_{1}^{*}$, with the differences being quite dramatic. This is perhaps not surprising given the undersizing that $\tilde{\xi}_{1}^{*}$ exhibits when breaks occur under the null. Comparing $\xi_{1}(\hat{\lambda})^{*}$ with the tests using unknown break date critical values, it is reassuring to see that the power losses associated with this test relative to $\tilde{\xi}_{1}$ and $\xi_{1}(\hat{\lambda})$ are not too severe. These losses become less marked the larger the break magnitude, and are less severe when a break occurs in the middle of the series; indeed, when $\lambda^{\prime}=0.5, \xi_{1}(\hat{\lambda})^{*}$ outperforms $\xi_{1}(\hat{\lambda})$ for all non-zero breaks, and $\tilde{\xi}_{1}$ for large breaks. Thus the cost in terms of power of employing a test with reliable size is relatively small in most circumstances.

With regard to the smooth transition tests, there is little to separate $s_{1}$ and $s_{1}^{*}$ : neither test has uniformly greater power when $\sigma_{\eta}^{2}=0.01,0.1$, although $s_{1}^{*}$ does have some power advantage when $\sigma_{\eta}^{2}=1$. Given the size problems associated with $s_{1}$ when a break occurs under the null, these results confirm the value of the $s_{1}^{*}$ version of the test when a break is actually present. Both tests have lower power than the instant break tests (with the exception of $\tilde{\xi}_{1}$ ), and the differences are more marked for small break magnitudes than for large. Some power loss is expected due to the additional generality which the smooth transition tests provide, but given the potential advantages of this testing approach, the power losses are not too severe, particularly when compared to the reliably sized $\xi_{1}(\hat{\lambda})^{*}$ test.

\subsection{Size and Power when a Smooth Transition Occurs}

Finally, we examine the sizes of the tests when a smooth transition occurs under the null hypothesis. We consider two sets of comparisons: one where a transition in level occurs and one where the transition is in both level and trend. We use Model $2 a$ in preference to Model 1 for the first set of simulations, since stationarity about a slow transition in level can appear like a trend stationary process, so that application of the Model 1 versions of the instant break tests would lead to inappropriate comparisons. For the latter set of experiments we use Model 2. The generating processes for these simulations are given by (12) and (11) respectively with $\sigma_{\eta}^{2}=0, \mu_{0}=\beta=0$ and $\varepsilon_{t} \sim \operatorname{NID}(0,1)$. For Model 2, the 
level and trend components of the breaks are isolated by adjusting the magnitude of $\delta_{\mu}$ to account for the change in intercept implicit in a break in trend. This adjustment is made using the transition midpoint as a reference, thus $\delta_{\mu}=\theta-\delta_{\beta} \lambda^{\prime} T$ with $\theta=0,2.5,5,10$. The results for all six tests for a range of transition speeds, timings and magnitudes are provided in Table VII.

The size results vary considerably depending on the magnitude and speed of the smooth transition. The instant break tests spuriously reject the null hypothesis very frequently for large transitions under both Models $2 a$ and 2. This oversize is generally less severe when the transitions have smaller magnitude, and for a given magnitude the tests generally suffer less distortion when the transition is fast $\left(\gamma^{\prime}=0.05\right.$ for Model $2 a$ being an exception since the series here closely approximates a linear trend). The only times the instant break tests reliably achieve close to correct size are when $\xi_{i}(\hat{\lambda})^{*}$ is applied to series which have medium to fast transitions of small magnitude, or when the generated series is close in appearance to trend stationarity $\left(\gamma^{\prime}=0.05\right.$ for Model 2a, again when the magnitude is small). In general the picture is one of severe oversizing when smooth transitions of typical speed and magnitude occur under the null.

In contrast, the sizes of the smooth transition test are not so volatile. The $s_{i}^{*}$ test exhibits reasonable size behaviour for medium to fast transitions-being just a little undersized-although for slower transitions, this feature of undersizing can become quite severe. The $s_{i}$ test is less reliable again, featuring moderate undersizing or oversizing in almost every case. It appears therefore that $s_{i}^{*}$ is the only test that has, irrespective of the magnitude of the structural change, reasonable properties for models with moderate to fast transitions. On the other hand, none of the tests can be considered satisfactory for models involving slower transitions, unless the rare situation arises of a very slow transition in level that is similar in appearance to a linear trend.

Regarding power comparisons when a smooth transition occurs under the alternative hypothesis, the instant break tests will always have a tendency to outperform the smooth transition tests. This follows in part from the former being oversized when smooth transitions occur under the null, but also from the way the stationarity tests are conducted. The tests are based on testing for stationarity in the residuals from a fitted deterministic model. Thus, if the true process is unit root with a smooth transition, the residuals from an instant break model will generally appear less stationary than those from a smooth 
transition model, and so the instant break tests will tend to reject more often. The main concern with stationarity testing when smooth transitions in linear trend are present is one of size behaviour, as analysed above.

\section{EMPIRICAL APPLICATIONS}

To illustrate the application of these tests, we investigate the trend behaviour of four economic time series. These are the end-year value of the S\&SP 500 stock market index for the period 1870-2000, annual UK gross domestic product from 1855-1913, the annual British index of industrial production from 1700-1913, and end-month observations on the dollar-sterling exchange rate from 1988:1-2001:12. The logarithms of the first three series are analysed, but no transformation is made on the exchange rate.

The stationarity tests in this paper are applied to the series, with the exception of $\tilde{\xi}_{i}^{*}$ due to the test's very poor size and power performance when structural change is present. In order to account for potential residual autocorrelation when conducting the tests, the KPSS nonparametric modification was employed, i.e. $\hat{\sigma}^{2}$ in the test statistics was replaced with $s^{2}(l)$ where

$$
s^{2}(l)=T^{-1} \sum_{t=1}^{T} e_{t}^{2}+2 T^{-1} \sum_{j=1}^{l} w(j, l) \sum_{t=j+1}^{T} e_{t} e_{t-j}
$$

Following KPSS, we use the Bartlett window for the weighting function: $w(j, l)=1-$ $j /(l+1)$, with the lag truncation parameter chosen by $l=\left[4(T / 100)^{1 / 4}\right]$ where [.] denotes integer part (see, for example, Schwert, 1989). Critical values for the tests using this KPSS correction were generated by simulation.

The $S \& P P 50$ series is an update of that used by Perron (1989) in his seminal study of testing for stationarity in the presence of trend breaks, in which he was able to reject the null of a unit root in favour of stationary deviations about a trend function with breaks in both level and slope at 1929. The results of applying the various tests to the four possible trend break models are shown in Panel A of Table VIII. For Models 1 and $2 a$, there is a clear rejection of stationarity (Model 1) or trend stationarity (Model 2a) around an instantaneous break in level, but no rejection when a smooth transition in level is assumed. A glance at the first and second rows of Figure 1, which superimposes each of the fitted trends in turn onto the observed series, easily explains these results. The simple instant level break models are clearly inappropriate trend specifications, so that the deviations from such trends are obviously nonstationary. The smooth transition 
models, on the other hand, provide much better fits to the series, and do not allow a rejection of the stationary null. However, this is because the parameter estimates imply extremely slow transitions with a midpoint estimated to be well outside of the sample period, so that what is being fit is basically equivalent to a low order polynomial in time! The piecewise trend Model $2 b$ also does not allow rejection of the null, with the tests being almost identical in selecting the break point to be in the mid-1940s (see the third row of Figure 1). Allowing both trend and level shifts also provides little evidence against the null, with only a rejection at the $10 \%$-level using the preferred $\xi_{2}(\hat{\lambda}), \xi_{2}(\hat{\lambda}) *$ tests allowing for an instant trend and level break, and a similar result when a smooth transition in level and trend is assumed. The strong rejection implied by $\tilde{\xi}_{2}$ is most likely due to the substantial oversizing that the test displays for breaks of this magnitude, as observed in the simulations. We would thus conclude that, if we are to model the $S \& P 500$ index as stationary deviations about a deterministic trend, then a formulation allowing an evolving trend is necessary, as found by Perron (1989) for a sample period ending in 1970. Our examination of a longer sample places the break date rather later than the 1929 assumed by Perron.

The tests for UK output are reported in Panel B, and show clear rejections for all models except Model 2, indicating that the series can be modelled as stationary about a change in level and trend. Some interesting features emerge from these models. Allowing shifts in both level and trend fits the break at 1919, with the corresponding smooth transition having a rapid transition with a midpoint also during 1919. The underlying models estimate the pre- and post-break trend growth rates to be $1.9 \%$ and $2.2 \%$ per annum respectively, and these are consistent with a precursor model presented in Mills (1994). Model $2 a$ restricts the pre- and post-break trend growths to be identical, allowing only level shifts in output. It is clear from the second row of Figure 2 that break date estimation using criterion (8) provides a much more plausible trend function than that suggested by criterion (7), illustrating the general superiority of (8) over (7). The imposition of the pre- and post-break trend growth restriction, which is difficult to detect visually in Figure 2 but is statistically rejected in Model 2, is responsible for the rejection of stationarity in Model $2 a$. This underlines the sensitivity of the tests to the correct trend specification.

Panel $\mathrm{C}$ reports the results for the index of industrial production, whose trend evo- 
lution has been analysed in the context of the industrial revolution by Mills and Crafts (1996) and Crafts and Mills (1997). In the latter, a Model 2-type smooth transition was fitted after a rejection of the unit root null hypothesis had been found using the Leybourne et al. (1998) testing procedure. The $s_{2}$ and $s_{2}^{*}$ tests are in agreement with this view that deviations in industrial production from a smooth transition in linear trend are stationary, while all the instantaneous break tests reject stationarity; these models have obviously inferior fits, as can be seen in Figure 3.

Lastly, Panel D reports the test statistics for the dollar-sterling exchange rate. The sample period was chosen to start after a period of adjustment following the Louvre Accord in March 1987. Here the test statistics follow a rather different pattern to those of the previous examples. Clear rejections are found for all statistics except the preferred instant break and smooth transition versions for Model 1. The reasons for this are again made clear by Figure 4. All models containing trends clearly lead to unrealistic trend functions and nonstationary residuals. In addition, Model 1 where the break date is estimated using (7) also results in an unrealistic trend function, placing the break somewhat later than that implied by (8) and the smooth transition model, again confirming the relative advantage of (8) over (7). The acceptable statistics are thus for those models that embody just an instantaneous level shift, or equivalently, an extremely fast smooth transition in mean. The break point is identified to be at September 1992, when the UK abruptly left the European Exchange Rate Mechanism (ERM). It would thus seem that the dollarsterling exchange rate might be characterised as stationary deviations about a constant mean that underwent an abrupt downward shift in response to a regime change. However, investigation of the stochastic properties of the deviations in the two regimes offers further insight into the behaviour of this exchange rate. During the ERM regime, the deviations are characterised by a second order autoregressive process with complex roots of $0.62 \pm$ $0.10 i$, thus producing the 'long swings' around a constant mean of 1.76 observed in the data. Although the ERM did not set a band for the dollar-sterling exchange rate, it seems to have constrained the fluctuations that this rate could take. The process for the post-ERM period is best represented by a first order autoregression with a root of 0.92 : in other words, it is close to the driftless random walk that is usually found to characterise freely floating exchange rate regimes, perhaps giving rise to the rejections at the $10 \%$-level observed for $\xi_{1}(\hat{\lambda})^{*}$ and $s_{1}^{*}$. 


\section{CONCLUSION}

We have examined several tests of the null hypothesis of stationarity against a unit root alternative where the series concerned admits structural change at an unknown point in time. Four tests are considered which permit an instantaneous break in level and/or trend, and two which allow a more general deterministic structure where the series undergoes a smooth transition between two different level and/or trend regimes. The tests have been applied to four economic time series.

The instant break tests rely, at least implicitly, on some method for estimating the unknown break date. The simulation results show clearly that break date estimation based on the significance of relevant dummy variable coefficients (or, equivalently, minimum sum of squared residuals or SBC) consistently outperforms Zivot-Andrews-type approaches where the break date is selected to give most weight to the null of (trend) stationarity about a break. This then has implications for the empirical sizes of tests based on the respective approaches when a break occurs under the null, with the Zivot-Andrews-based tests proving to be inferior in terms of size distortion to the equivalent tests based on dummy variable coefficient significance.

The instant break test which has best overall size behaviour uses dummy variable coefficient significance to estimate the break date, and employs critical values appropriate for tests where the break date is known, on the basis that the correct break date will be selected superconsistently; this procedure coincides with that proposed by Busetti

and Harvey (2002) and Lee (1999). This preferred test has less power than alternative procedures in some circumstances, but generally the cost in terms of foregone power from using such a reliably sized test is small. A caveat to this recommendation occurs when no break actually occurs in the series. In this case the preferred test is invalid, and suffers from substantial undersizing and low power. Busetti and Harvey (2002) argue that if uncertainty exists concerning the presence of a break, their Zivot-Andrews-type test should instead be used, which employs unknown break date critical values consistent with an assumption that the breaks have small magnitude. On the basis of our results, we would modify this recommendation to suggest use of the newly proposed variant of this test, where the test is based on a dummy variable coefficient break date estimator, but the same small break assumption is maintained for determining the critical values. This follows since, if a small break does occur in the series, the size distortions are less severe 
for the latter test. Our recommendation is therefore always to use tests where the break date is selected on the basis of dummy variable coefficient significance, but modifying the critical values depending on the degree of confidence in the existence of a break. This approach is also appealing in the sense that break date selection is then always consistent with the preferred method in the parallel context of testing the unit root null against a stationary alternative.

The new smooth transition tests provide additional generality in the stationarity testing procedure. The preferred version of the test, which uses critical values associated with tests where the transition midpoint and speed are known, has good size properties and reasonable power when an instant break occurs under the null, and has greatly superior size performance compared to the instant break tests if the null is of stationarity around a moderate to fast smooth transition. The limitations of the procedure are when the transition speed is slow or when no break or transition occurs, although in the latter scenario the smooth transition test which uses critical values for which the transition midpoint and speed are unknown has correct size by construction. Given that structural change in economic time series often occurs gradually rather than instantaneously, these smooth transition tests have potential value in practical applications and provide a useful stationarity testing counterpart to the unit root tests proposed by Leybourne et al. (1998).

\section{APPENDIX}

Proof of (9): In order to show that the asymptotic distribution of $\xi_{i}(\hat{\lambda})(i=1,2,2 a, 2 b)$ is as given in (9), it is sufficient to show that the limiting distributions of $t_{\hat{\delta}_{\mu}}$ for Models 1 and $2 a, t_{\hat{\delta}_{\beta}}$ for Model $2 b$, and $F_{\hat{\delta}, \hat{\beta}}$ for Model 2, are given by $Z_{i}(\lambda)(i=1,2 a, 2 b, 2)$ respectively. The result then follows, using the established asymptotic results of Busetti and Harvey (2001) and Harvey and Mills (2002) for the stationarity test statistic with a given break fraction.

Consider first Model 1 (proofs for Models $2 a$ and $2 b$ follow with appropriate modifications and are omitted). Under the null of stationarity $\left(\sigma_{\eta}^{2}=0\right)$ about a break in level of magnitude $\delta_{\mu}$ at time $\tau^{\prime}$, the generating process can be written as follows, assuming $\mu_{0}=0$ without loss of generality:

$$
y_{t}=\delta_{\mu} w_{t}^{\prime}+\varepsilon_{t}
$$


The appropriate regression for this model with an assumed break date $\tau$ is then

$$
y_{t}=\mu+\delta_{\mu} w_{t}+\varepsilon_{t}
$$

with the OLS estimator of $\delta_{\mu}$ given by

$$
\hat{\delta}_{\mu}=(T-\tau)^{-1} \sum_{t=\tau+1}^{T} y_{t}-\tau^{-1} \sum_{t=1}^{\tau} y_{t}
$$

Substituting in for $y_{t}$ and scaling appropriately yields

$$
T^{1 / 2} \hat{\delta}_{\mu}=T^{1 / 2} \delta_{\mu}\left[\frac{1-\lambda^{\prime}}{1-\lambda}-I \frac{\lambda-\lambda^{\prime}}{\lambda(1-\lambda)}\right]+T^{-1 / 2}(1-\lambda)^{-1} \sum_{t=\tau+1}^{T} \varepsilon_{t}-T^{-1 / 2} \lambda^{-1} \sum_{t=1}^{\tau} \varepsilon_{t}
$$

where $I=1\left(\tau>\tau^{\prime}\right)$ and $1($.$) is the indicator function. Now the shift assumption can be$ represented as $\delta_{\mu}=k T^{-\left(\kappa+\frac{1}{2}\right)}$ where $\kappa>0$. Use of this representation, combined with the results of Lemma 2 in Busetti and Harvey (2001), leads to the asymptotic distribution of $\hat{\delta}_{\mu}$ :

$$
\sigma^{-1} T^{1 / 2} \hat{\delta}_{\mu} \Rightarrow(1-\lambda)^{-1}[W(1)-W(\lambda)]-\lambda^{-1} W(\lambda)
$$

The estimated variance of $\hat{\delta}_{\mu}$ is given by

$$
\hat{\sigma}_{\hat{\delta}_{\mu}}^{2}=\hat{\sigma}^{2} \tau^{-1}(T-\tau)^{-1} T
$$

Scaling, and noting that $\hat{\sigma}^{2} \stackrel{p}{\longrightarrow} \sigma^{2}$, gives the limiting distribution of this estimator:

$$
T \hat{\sigma}_{\hat{\delta}_{\mu}}^{2} \Rightarrow \sigma^{2} \lambda^{-1}(1-\lambda)^{-1}
$$

The asymptotic distribution of $t_{\hat{\delta}_{\mu}}=\hat{\delta}_{\mu} / \hat{\sigma}_{\hat{\delta}_{\mu}}$ then follows:

$$
t_{\hat{\delta}_{\mu}} \Rightarrow \sqrt{\frac{\lambda}{1-\lambda}}\left[W(1)-\frac{1}{\lambda} W(\lambda)\right]
$$

Consider now Model 2. The generating process under the null can be expressed as follows, assuming $\mu_{0}=0$ and $\beta=0$ without loss of generality:

$$
y_{t}=\delta_{\mu} w_{t}^{\prime}+\delta_{\beta}\left(w_{t}^{\prime} t\right)+\varepsilon_{t}
$$

The appropriate regression with an assumed break date $\tau$ is now

$$
y_{t}=\mu+\beta t+\delta_{\mu} w_{t}+\delta_{\beta}\left(w_{t} t\right)+\varepsilon_{t}
$$


with the (scaled) OLS estimators of the regression parameters given by

$$
\left[\begin{array}{c}
T^{1 / 2} \hat{\mu} \\
T^{3 / 2} \hat{\beta} \\
T^{1 / 2} \hat{\delta}_{\mu} \\
T^{3 / 2} \hat{\delta}_{\beta}
\end{array}\right] \approx\left[\begin{array}{cccc}
1 & \frac{1}{2} & 1-\lambda & \frac{1}{2}\left(1-\lambda^{2}\right) \\
\frac{1}{2} & \frac{1}{3} & \frac{1}{2}\left(1-\lambda^{2}\right) & \frac{1}{3}\left(1-\lambda^{3}\right) \\
1-\lambda & \frac{1}{2}\left(1-\lambda^{2}\right) & 1-\lambda & \frac{1}{2}\left(1-\lambda^{2}\right) \\
\frac{1}{2}\left(1-\lambda^{2}\right) & \frac{1}{3}\left(1-\lambda^{3}\right) & \frac{1}{2}\left(1-\lambda^{2}\right) & \frac{1}{3}\left(1-\lambda^{3}\right)
\end{array}\right]^{-1}\left[\begin{array}{c}
T^{-1 / 2} \sum_{t=1}^{T} y_{t} \\
T^{-3 / 2} \sum_{t=1}^{T} t y_{t} \\
T^{-1 / 2} \sum_{t=\tau+1}^{T} y_{t} \\
T^{-3 / 2} \sum_{t=\tau+1}^{T} t y_{t}
\end{array}\right]
$$

in large samples. Lemma 2 of Harvey and Busetti (2001) and the shift assumption, which here implies $\delta_{\mu}=k_{1} T^{-\left(\kappa_{1}+\frac{1}{2}\right)}$ and $\delta_{\beta}=k_{2} T^{-\left(\kappa_{2}+\frac{3}{2}\right)}$, where $\kappa_{1}, \kappa_{2}>0$, allows derivation of the limits of the final right hand side terms:

$$
\begin{aligned}
& T^{-1 / 2} \sum_{t=1}^{T} y_{t} \Rightarrow \sigma W(1) \\
& T^{-3 / 2} \sum_{t=1}^{T} t y_{t} \Rightarrow \sigma \int_{0}^{1} r d W(r) \\
& T^{-1 / 2} \sum_{t=\tau+1}^{T} y_{t} \Rightarrow \sigma[W(1)-W(\lambda)] \\
& T^{-3 / 2} \sum_{t=\tau+1}^{T} t y_{t} \Rightarrow \sigma \int_{\lambda}^{1} r d W(r)
\end{aligned}
$$

The asymptotic distributions of the relevant parameter estimates then follow:

$$
\begin{aligned}
& T^{1 / 2} \hat{\delta}_{\mu} \Rightarrow 2 \sigma \lambda^{-2}(1-\lambda)^{-3} H_{1} \\
& T^{3 / 2} \hat{\delta}_{\beta} \Rightarrow 6 \sigma \lambda^{-3}(1-\lambda)^{-3} H_{2}
\end{aligned}
$$

where

$$
\begin{aligned}
H_{1}= & 2 \lambda^{2}\left(1+\lambda+\lambda^{2}\right) W(1)-2 \lambda\left(1-2 \lambda+4 \lambda^{2}\right) W(\lambda)+3(1-\lambda)^{3} \int_{0}^{\lambda} r d W(r) \\
& -3 \lambda^{2}(1+\lambda) \int_{\lambda}^{1} r d W(r) \\
H_{2}= & -\lambda^{3}(1+\lambda) W(1)+\lambda\left(1-3 \lambda+4 \lambda^{2}\right) W(\lambda)-2(1-\lambda)^{3} \int_{0}^{\lambda} r d W(r)+2 \lambda^{3} \int_{\lambda}^{1} r d W(r)
\end{aligned}
$$

Now $F_{\hat{\delta}, \hat{\beta}}=(R b)^{\prime}\left[R\left(X^{\prime} X\right)^{-1} R^{\prime}\right]^{-1}(R b) / 2 \hat{\sigma}^{2}$ where $b$ is the vector of OLS parameter estimates, $X$ is the matrix of regressors, and

$$
R=\left[\begin{array}{llll}
0 & 0 & 1 & 0 \\
0 & 0 & 0 & 1
\end{array}\right]
$$

This allows us to write

$$
F_{\hat{\delta}, \hat{\beta}} \approx\left[\begin{array}{ll}
T^{1 / 2} \hat{\delta}_{\mu} & T^{3 / 2} \hat{\delta}_{\beta}
\end{array}\right]\left[\begin{array}{cc}
\frac{4\left(1-2 \lambda+4 \lambda^{2}\right)}{\lambda(1-\lambda)^{3}} & \frac{-6\left(1-3 \lambda+4 \lambda^{2}\right)}{\lambda^{2}(1-\lambda)^{3}} \\
\frac{-6\left(1-3 \lambda+4 \lambda^{2}\right)}{\lambda^{2}(1-\lambda)^{3}} & \frac{12\left(1-3 \lambda+3 \lambda^{2}\right)}{\lambda^{3}(1-\lambda)^{3}}
\end{array}\right]^{-1}\left[\begin{array}{c}
T^{1 / 2} \hat{\delta}_{\mu} \\
T^{3 / 2} \hat{\delta}_{\beta}
\end{array}\right] / 2 \hat{\sigma}^{2}
$$

Given $\hat{\sigma}^{2} \stackrel{p}{\longrightarrow} \sigma^{2}$, the asymptotic distribution of $F_{\hat{\delta}, \hat{\beta}}$ can then be obtained:

$$
F_{\hat{\delta}, \hat{\beta}} \Rightarrow \frac{2}{\lambda^{3}(1-\lambda)^{5}}\left[\left(1-3 \lambda+3 \lambda^{2}\right) H_{1}^{2}+3\left(1-3 \lambda+4 \lambda^{2}\right)^{2} H_{1} H_{2}+3\left(1-2 \lambda+4 \lambda^{2}\right) H_{2}^{2}\right]
$$




\section{REFERENCES}

Bacon DW, Watts, DG. 1971. Estimating the transition between two intersecting straight lines. Biometrika 58: 525-534.

Busetti F, Harvey AC. 2001. Testing for the presence of a random walk in series with structural breaks. Journal of Time Series Analysis 22: 127-150.

Busetti F, Harvey AC. 2002. Further comments on stationarity tests in series with structural breaks at unknown points. Discussion Paper, Bank of Italy and University of Cambridge.

Crafts NFR, Mills TC. 1997. Endogenous innovation, trend growth and the British industrial revolution. Journal of Economic History 57: 950-956.

Dickey DA, Fuller WA. 1979. Distribution of the estimators for autoregressive time series with a unit root. Journal of the American Statistical Association 73: 427-431.

Granger CWJ, Teräsvirta T. 1993. Modelling Nonlinear Economic Relationships, Oxford University Press: Oxford.

Harvey DI, Leybourne SJ, Newbold P. 2001. Innovational outlier unit root tests with an endogenously determined break in level. Oxford Bulletin of Economics and Statistics 63: $559-575$.

Harvey DI, Mills TC. (2002). A note on Busetti-Harvey tests for stationarity in series with structural breaks. Journal of Time Series Analysis, forthcoming.

Kwiatkowski D, Phillips PCB, Schmidt P, Shin Y. 1992. Testing the null hypothesis of stationarity against the alternative of a unit root: how sure are we that economic time series have a unit root? Journal of Econometrics 54: 159-178.

Lee J. 1999. Stationarity tests with multiple endogenized breaks. In Nonlinear Time Series Analysis of Economic and Financial Data, Rothman P (ed). Kluwer Academic Press: Massachusetts.

Lee J, Strazicich M. 2001. Testing the null of stationarity in the presence of a structural break. Applied Economics Letters 8: 377-382. 
Leybourne SJ, McCabe BPM. 1994. A consistent test for a unit root. Journal of Business and Economic Statistics 12: 157-166.

Leybourne SJ, McCabe BPM. 1999. Modified stationarity tests with data-dependent model-selection rules. Journal of Business and Economic Statistics 17: 264-270.

Leybourne SJ, Newbold P, Vougas D. 1998. Unit roots and smooth transitions. Journal of Time Series Analysis 19: 83-97.

Lin CJ, Teräsvirta T. 1994. Testing the constancy of regression parameters against continuous structural change. Journal of Econometrics 62: 211-228.

MacNeill IB. 1978. Properties of sequences of partial sums of polynomial regression residuals with applications to tests for change of regression at unknown times. Annals of Statistics 6: 422-433.

Mills TC. 1994. Segmented trends and the stochastic properties of UK output. Applied Economics Letters 1: 132-133.

Mills TC, Crafts NFR. 1996. Trend growth in British industrial output, 1700-1913: A reappraisal. Explorations in Economic History 33: 277-295.

Nyblom J, Mäkeläinen T. 1983. Comparisons of tests for the presence of random walk coefficients in a simple linear model. Journal of the American Statistical Association 78: $856-864$.

Perron P. 1989. The Great Crash, the oil price shock, and the unit root hypothesis. Econometrica 57: 1361-1401.

Perron P. 1993. The Great Crash, the oil price shock, and the unit root hypothesis: Erratum. Econometrica 61: 248-249.

Schwert GW. 1989. Tests for unit roots: A Monte Carlo investigation. Journal of Business and Economic Statistics 7: 147-159.

Vogelsang TJ, Perron P. 1998. Additional tests for a unit root allowing for a break in the trend function at an unknown time. International Economic Review 39: 1073-1100.

Zivot E, Andrews DWK. 1992. Further evidence on the Great Crash, the oil price shock, and the unit-root hypothesis. Journal of Business and Economic Statistics 10: 251-270. 
Table I. Critical values of $\xi_{i}(\hat{\lambda})$ tests for stationarity with an endogenously determined structural break

\begin{tabular}{lcccccccc}
\hline & \multicolumn{3}{c}{ Model 1 } & & \multicolumn{3}{c}{ Model 2 } \\
\cline { 2 - 4 } \cline { 7 - 8 } & $10 \%$ & $5 \%$ & $1 \%$ & & $10 \%$ & $5 \%$ & $1 \%$ \\
\hline$T=50$ & 0.167 & 0.209 & 0.311 & & 0.047 & 0.057 & 0.078 \\
$T=100$ & 0.170 & 0.212 & 0.320 & & 0.048 & 0.057 & 0.081 \\
$T=200$ & 0.177 & 0.219 & 0.323 & & 0.048 & 0.057 & 0.081 \\
$T=\infty$ & 0.177 & 0.221 & 0.326 & & 0.049 & 0.057 & 0.081 \\
\hline \multicolumn{4}{c}{} & \multicolumn{3}{c}{ Model $2 a$} & & \multicolumn{3}{c}{ Model $2 b$} \\
\cline { 2 - 4 } \cline { 6 - 8 } & $10 \%$ & $5 \%$ & $1 \%$ & & $10 \%$ & $5 \%$ & $1 \%$ \\
\hline$T=50$ & 0.070 & 0.085 & 0.122 & & 0.062 & 0.075 & 0.108 \\
$T=100$ & 0.071 & 0.086 & 0.119 & & 0.062 & 0.076 & 0.112 \\
$T=200$ & 0.072 & 0.086 & 0.122 & & 0.062 & 0.077 & 0.107 \\
$T=\infty$ & 0.073 & 0.088 & 0.122 & & 0.062 & 0.075 & 0.106 \\
\hline
\end{tabular}

Table II. Critical values of $s_{i}$ tests for stationarity with an endogenously determined smooth transition in linear trend

\begin{tabular}{lccccccccccc}
\hline & \multicolumn{3}{c}{ Model 1 } & & \multicolumn{3}{c}{ Model 2} & & \multicolumn{3}{c}{ Model $2 a$} \\
\cline { 2 - 3 } \cline { 10 - 12 } & $10 \%$ & $5 \%$ & $1 \%$ & & $10 \%$ & $5 \%$ & $1 \%$ & & $10 \%$ & $5 \%$ & $1 \%$ \\
\hline$T=50$ & 0.170 & 0.218 & 0.339 & & 0.049 & 0.059 & 0.084 & & 0.062 & 0.074 & 0.104 \\
$T=100$ & 0.175 & 0.225 & 0.331 & & 0.050 & 0.061 & 0.088 & & 0.063 & 0.077 & 0.105 \\
$T=200$ & 0.180 & 0.232 & 0.358 & & 0.051 & 0.062 & 0.086 & & 0.064 & 0.076 & 0.108 \\
$T=500$ & 0.181 & 0.229 & 0.346 & & 0.051 & 0.062 & 0.090 & & 0.066 & 0.079 & 0.110 \\
\hline
\end{tabular}


Table III. Critical values of $s_{i}$ tests for stationarity with a known smooth transition in linear trend: 5\%-level, $T=100$

\begin{tabular}{|c|c|c|c|c|c|c|}
\hline \multicolumn{7}{|c|}{ Panel A. Model 1} \\
\hline$\tau^{\prime}$ & $\gamma^{\prime}=0.05$ & $\gamma^{\prime}=0.10$ & $\gamma^{\prime}=0.25$ & $\gamma^{\prime}=0.50$ & $\gamma^{\prime}=1.00$ & $\gamma^{\prime}=2.00$ \\
\hline 0.01 & 0.191 & 0.275 & 0.373 & 0.404 & 0.428 & 0.441 \\
\hline 0.10 & 0.179 & 0.252 & 0.331 & 0.357 & 0.370 & 0.376 \\
\hline 0.20 & 0.167 & 0.218 & 0.272 & 0.290 & 0.296 & 0.302 \\
\hline 0.30 & 0.155 & 0.181 & 0.217 & 0.230 & 0.234 & 0.237 \\
\hline 0.40 & 0.147 & 0.156 & 0.177 & 0.188 & 0.195 & 0.197 \\
\hline 0.50 & 0.144 & 0.147 & 0.162 & 0.172 & 0.178 & 0.182 \\
\hline 0.60 & 0.147 & 0.155 & 0.175 & 0.185 & 0.192 & 0.196 \\
\hline 0.70 & 0.154 & 0.179 & 0.208 & 0.222 & 0.229 & 0.233 \\
\hline 0.80 & 0.166 & 0.211 & 0.259 & 0.278 & 0.287 & 0.291 \\
\hline 0.90 & 0.178 & 0.244 & 0.317 & 0.342 & 0.352 & 0.356 \\
\hline 0.99 & 0.189 & 0.270 & 0.364 & 0.404 & 0.425 & 0.434 \\
\hline \multicolumn{7}{|c|}{ Panel B. Model 2} \\
\hline$\tau^{\prime}$ & $\gamma^{\prime}=0.05$ & $\gamma^{\prime}=0.10$ & $\gamma^{\prime}=0.25$ & $\gamma^{\prime}=0.50$ & $\gamma^{\prime}=1.00$ & $\gamma^{\prime}=2.00$ \\
\hline 0.01 & 0.059 & 0.072 & 0.104 & 0.124 & 0.133 & 0.137 \\
\hline 0.10 & 0.058 & 0.068 & 0.096 & 0.112 & 0.118 & 0.120 \\
\hline 0.20 & 0.057 & 0.062 & 0.082 & 0.091 & 0.095 & 0.096 \\
\hline 0.30 & 0.056 & 0.058 & 0.068 & 0.074 & 0.076 & 0.077 \\
\hline 0.40 & 0.055 & 0.053 & 0.057 & 0.061 & 0.063 & 0.063 \\
\hline 0.50 & 0.055 & 0.052 & 0.053 & 0.056 & 0.058 & 0.059 \\
\hline 0.60 & 0.055 & 0.054 & 0.058 & 0.060 & 0.062 & 0.063 \\
\hline 0.70 & 0.056 & 0.057 & 0.066 & 0.072 & 0.074 & 0.075 \\
\hline 0.80 & 0.057 & 0.063 & 0.080 & 0.088 & 0.091 & 0.093 \\
\hline 0.90 & 0.058 & 0.068 & 0.093 & 0.110 & 0.116 & 0.117 \\
\hline 0.99 & 0.059 & 0.072 & 0.103 & 0.122 & 0.132 & 0.138 \\
\hline \multicolumn{7}{|c|}{ Panel C. Model $2 a$} \\
\hline$\tau^{\prime}$ & $\gamma^{\prime}=0.05$ & $\gamma^{\prime}=0.10$ & $\gamma^{\prime}=0.25$ & $\gamma^{\prime}=0.50$ & $\gamma^{\prime}=1.00$ & $\gamma^{\prime}=2.00$ \\
\hline 0.01 & 0.086 & 0.098 & 0.122 & 0.132 & 0.137 & 0.142 \\
\hline 0.10 & 0.084 & 0.092 & 0.108 & 0.116 & 0.119 & 0.120 \\
\hline 0.20 & 0.083 & 0.084 & 0.092 & 0.097 & 0.100 & 0.102 \\
\hline 0.30 & 0.085 & 0.083 & 0.087 & 0.094 & 0.097 & 0.099 \\
\hline 0.40 & 0.101 & 0.101 & 0.106 & 0.111 & 0.114 & 0.116 \\
\hline 0.50 & 0.128 & 0.127 & 0.128 & 0.130 & 0.131 & 0.132 \\
\hline 0.60 & 0.103 & 0.104 & 0.110 & 0.115 & 0.117 & 0.118 \\
\hline 0.70 & 0.086 & 0.083 & 0.088 & 0.094 & 0.098 & 0.100 \\
\hline 0.80 & 0.083 & 0.084 & 0.090 & 0.094 & 0.097 & 0.099 \\
\hline 0.90 & 0.084 & 0.091 & 0.107 & 0.115 & 0.117 & 0.118 \\
\hline 0.99 & 0.085 & 0.097 & 0.119 & 0.130 & 0.137 & 0.141 \\
\hline
\end{tabular}


Table IV. Empirical sizes of nominal 5\%-level stationarity tests when a structural break occurs under the null: $T=100$

\begin{tabular}{|c|c|c|c|c|c|c|c|c|}
\hline \multicolumn{9}{|c|}{ Panel A. Model 1} \\
\hline$\delta_{\beta}$ & $\delta_{\mu}$ & $\lambda^{\prime}$ & $\tilde{\xi}_{1}$ & $\tilde{\xi}_{1}^{*}$ & $\xi_{1}(\hat{\lambda})$ & $\xi_{1}(\hat{\lambda})^{*}$ & $s_{1}$ & $s_{1}^{*}$ \\
\hline \multirow[t]{10}{*}{-} & 0.0 & - & 5.08 & 0.07 & 5.01 & 3.70 & 5.00 & 2.54 \\
\hline & 2.5 & 0.25 & 17.87 & 1.46 & 9.21 & 4.73 & 6.04 & 3.70 \\
\hline & & 0.50 & 10.28 & 1.31 & 2.71 & 4.84 & 1.49 & 3.93 \\
\hline & & 0.75 & 17.55 & 1.32 & 9.02 & 4.89 & 6.64 & 4.49 \\
\hline & 5.0 & 0.25 & 17.62 & 1.30 & 9.72 & 4.94 & 7.50 & 4.52 \\
\hline & & 0.50 & 10.46 & 1.48 & 2.77 & 4.98 & 2.21 & 4.92 \\
\hline & & 0.75 & 17.45 & 1.23 & 9.09 & 5.03 & 7.76 & 5.29 \\
\hline & 10.0 & 0.25 & 14.81 & 0.37 & 9.78 & 4.99 & 8.16 & 4.88 \\
\hline & & 0.50 & 9.92 & 1.34 & 2.76 & 4.96 & 2.12 & 5.15 \\
\hline & & 0.75 & 14.51 & 0.34 & 9.09 & 5.07 & 7.66 & 5.05 \\
\hline \multicolumn{9}{|c|}{ Panel B. Model $2 a$} \\
\hline$\delta_{\beta}$ & $\delta_{\mu}$ & $\lambda^{\prime}$ & $\tilde{\xi}_{2 a}$ & $\tilde{\xi}_{2 a}^{*}$ & $\xi_{2 a}(\hat{\lambda})$ & $\xi_{2 a}(\hat{\lambda})^{*}$ & $s_{2 a}$ & $s_{2 a}^{*}$ \\
\hline \multirow[t]{10}{*}{-} & 0.0 & - & 5.08 & 0.03 & 4.81 & 1.51 & 4.87 & 0.77 \\
\hline & 2.5 & 0.25 & 21.97 & 1.79 & 8.83 & 4.73 & 8.65 & 2.82 \\
\hline & & 0.50 & 23.83 & 0.58 & 16.34 & 4.86 & 17.33 & 3.70 \\
\hline & & 0.75 & 22.70 & 1.56 & 8.62 & 4.72 & 8.77 & 3.47 \\
\hline & 5.0 & 0.25 & 22.42 & 1.85 & 9.01 & 4.96 & 11.94 & 4.45 \\
\hline & & 0.50 & 22.29 & 0.25 & 16.78 & 5.02 & 20.33 & 4.47 \\
\hline & & 0.75 & 22.17 & 1.77 & 8.79 & 4.93 & 12.00 & 4.63 \\
\hline & 10.0 & 0.25 & 23.02 & 1.61 & 9.05 & 4.98 & 12.08 & 4.95 \\
\hline & & 0.50 & 22.83 & 0.02 & 16.82 & 5.01 & 20.27 & 4.89 \\
\hline & & 0.75 & 23.25 & 1.57 & 8.77 & 4.94 & 12.44 & 5.05 \\
\hline \multicolumn{9}{|c|}{ Panel C. Model $2 b$} \\
\hline$\delta_{\beta}$ & $\delta_{\mu}$ & $\lambda^{\prime}$ & $\tilde{\xi}_{2 b}$ & $\tilde{\xi}_{2 b}^{*}$ & $\xi_{2 b}(\hat{\lambda})$ & $\xi_{2 b}(\hat{\lambda})^{*}$ & $s_{2}$ & $s_{2}^{*}$ \\
\hline 0 & - & - & 5.14 & 0.58 & 5.00 & 1.55 & 5.03 & 0.84 \\
\hline \multirow[t]{3}{*}{1} & - & 0.25 & 7.67 & 0.90 & 9.90 & 3.43 & 9.50 & 3.14 \\
\hline & & 0.50 & 2.52 & 0.49 & 3.25 & 2.07 & 3.02 & 3.54 \\
\hline & & 0.75 & 7.41 & 0.94 & 9.32 & 3.41 & 8.67 & 3.19 \\
\hline \multirow[t]{3}{*}{2} & - & 0.25 & 11.98 & 1.41 & 12.51 & 4.63 & 13.28 & 4.44 \\
\hline & & 0.50 & 9.64 & 2.69 & 6.61 & 4.67 & 3.49 & 4.23 \\
\hline & & 0.75 & 12.25 & 1.51 & 11.70 & 4.58 & 12.67 & 4.56 \\
\hline \multirow[t]{3}{*}{4} & - & 0.25 & 19.07 & 4.99 & 13.21 & 5.12 & 16.05 & 5.68 \\
\hline & & 0.50 & 11.57 & 4.98 & 6.93 & 4.98 & 3.71 & 4.13 \\
\hline & & 0.75 & 18.16 & 4.87 & 12.20 & 4.96 & 15.77 & 5.87 \\
\hline \multicolumn{9}{|c|}{ Panel D. Model 2} \\
\hline$\delta_{\beta}$ & $\delta_{\mu}$ & $\lambda^{\prime}$ & $\tilde{\xi}_{2}$ & $\tilde{\xi}_{2}^{*}$ & $\xi_{2}(\hat{\lambda})$ & $\xi_{2}(\hat{\lambda})^{*}$ & $s_{2}$ & $s_{2}^{*}$ \\
\hline \multirow[t]{4}{*}{0} & 0.0 & - & 4.67 & 0.03 & 5.07 & 1.15 & 5.03 & 0.84 \\
\hline & 2.5 & 0.25 & 26.84 & 1.73 & 17.30 & 4.54 & 10.49 & 3.08 \\
\hline & & 0.50 & 17.31 & 1.60 & 6.04 & 4.23 & 3.18 & 3.56 \\
\hline & & 0.75 & 27.61 & 1.64 & 17.80 & 4.23 & 10.57 & 2.90 \\
\hline
\end{tabular}




\begin{tabular}{|c|c|c|c|c|c|c|c|c|}
\hline \multicolumn{9}{|c|}{ Panel D. Model 2 contd. } \\
\hline$\delta_{\beta}$ & $\delta_{\mu}$ & $\lambda^{\prime}$ & $\tilde{\xi}_{2}$ & $\tilde{\xi}_{2}^{*}$ & $\xi_{2}(\hat{\lambda})$ & $\xi_{2}(\hat{\lambda})^{*}$ & $s_{2}$ & $s_{2}^{*}$ \\
\hline \multirow[t]{6}{*}{0} & 5.0 & 0.25 & 30.92 & 1.60 & 18.54 & 4.88 & 14.82 & 4.54 \\
\hline & & 0.50 & 17.45 & 1.70 & 6.48 & 4.55 & 3.89 & 4.32 \\
\hline & & 0.75 & 31.34 & 1.49 & 18.89 & 4.72 & 15.06 & 4.49 \\
\hline & 10.0 & 0.25 & 28.82 & 0.35 & 18.62 & 4.93 & 15.32 & 4.85 \\
\hline & & 0.50 & 18.52 & 1.52 & 6.54 & 4.59 & 4.22 & 4.48 \\
\hline & & 0.75 & 29.41 & 0.37 & 18.95 & 4.73 & 15.58 & 4.58 \\
\hline \multirow[t]{12}{*}{1} & 0.0 & 0.25 & 34.03 & 1.96 & 19.22 & 4.87 & 9.50 & 3.14 \\
\hline & & 0.50 & 18.80 & 1.87 & 6.48 & 4.61 & 3.02 & 3.54 \\
\hline & & 0.75 & 32.90 & 1.86 & 18.38 & 4.31 & 8.67 & 3.19 \\
\hline & 2.5 & 0.25 & 31.77 & 1.89 & 18.86 & 4.92 & 13.38 & 4.20 \\
\hline & & 0.50 & 14.20 & 0.78 & 6.36 & 4.50 & 3.03 & 3.39 \\
\hline & & 0.75 & 27.55 & 1.37 & 18.33 & 4.54 & 11.36 & 3.25 \\
\hline & 5.0 & 0.25 & 30.49 & 1.21 & 18.63 & 4.91 & 14.85 & 4.80 \\
\hline & & 0.50 & 15.66 & 1.01 & 6.47 & 4.54 & 4.02 & 4.38 \\
\hline & & 0.75 & 26.22 & 0.94 & 18.87 & 4.71 & 14.67 & 4.34 \\
\hline & 10.0 & 0.25 & 27.93 & 0.27 & 18.62 & 4.93 & 15.34 & 4.85 \\
\hline & & 0.50 & 18.86 & 1.51 & 6.54 & 4.59 & 4.45 & 4.71 \\
\hline & & 0.75 & 30.02 & 0.50 & 18.95 & 4.73 & 15.45 & 4.48 \\
\hline \multirow[t]{12}{*}{2} & 0.0 & 0.25 & 33.96 & 1.68 & 18.91 & 4.82 & 13.28 & 4.44 \\
\hline & & 0.50 & 18.76 & 1.87 & 6.29 & 4.52 & 3.49 & 4.23 \\
\hline & & 0.75 & 32.60 & 1.65 & 18.29 & 4.63 & 12.67 & 4.56 \\
\hline & 2.5 & 0.25 & 28.70 & 0.93 & 18.70 & 4.91 & 13.75 & 4.21 \\
\hline & & 0.50 & 13.56 & 0.69 & 6.33 & 4.55 & 3.24 & 3.65 \\
\hline & & 0.75 & 27.81 & 1.24 & 18.55 & 4.68 & 12.37 & 3.49 \\
\hline & 5.0 & 0.25 & 26.83 & 0.82 & 18.66 & 4.91 & 14.99 & 4.49 \\
\hline & & 0.50 & 11.02 & 0.20 & 6.49 & 4.56 & 4.44 & 4.73 \\
\hline & & 0.75 & 21.94 & 0.55 & 18.84 & 4.72 & 15.13 & 4.74 \\
\hline & 10.0 & 0.25 & 26.69 & 0.15 & 18.62 & 4.93 & 15.98 & 5.24 \\
\hline & & 0.50 & 19.00 & 1.13 & 6.54 & 4.59 & 4.67 & 4.91 \\
\hline & & 0.75 & 27.61 & 0.14 & 18.95 & 4.73 & 15.20 & 4.88 \\
\hline \multirow[t]{12}{*}{4} & 0.0 & 0.25 & 33.05 & 1.19 & 19.17 & 4.79 & 16.05 & 5.68 \\
\hline & & 0.50 & 18.12 & 1.68 & 6.51 & 4.76 & 3.71 & 4.13 \\
\hline & & 0.75 & 31.89 & 1.33 & 18.53 & 4.65 & 15.77 & 5.87 \\
\hline & 2.5 & 0.25 & 26.45 & 0.58 & 18.83 & 4.98 & 14.53 & 4.68 \\
\hline & & 0.50 & 12.54 & 0.66 & 6.40 & 4.46 & 3.48 & 3.77 \\
\hline & & 0.75 & 26.36 & 0.80 & 18.70 & 4.68 & 14.15 & 4.39 \\
\hline & 5.0 & 0.25 & 18.07 & 0.18 & 18.65 & 4.91 & 15.29 & 4.81 \\
\hline & & 0.50 & 6.82 & 0.09 & 6.46 & 4.54 & 3.82 & 4.22 \\
\hline & & 0.75 & 18.90 & 0.33 & 18.88 & 4.71 & 15.14 & 4.50 \\
\hline & 10.0 & 0.25 & 18.08 & 0.05 & 18.62 & 4.93 & 15.14 & 4.72 \\
\hline & & 0.50 & 7.49 & 0.00 & 6.54 & 4.59 & 4.47 & 4.87 \\
\hline & & 0.75 & 7.53 & 0.00 & 18.95 & 4.73 & 15.61 & 4.68 \\
\hline
\end{tabular}


Table V. Percentage of times the true break date is selected by estimation criteria (7) and (8) when a structural break occurs under the null: $T=100$

\begin{tabular}{|c|c|c|c|c|c|c|c|}
\hline \multicolumn{8}{|c|}{ Panel A. Model 1} \\
\hline \multirow[b]{2}{*}{$\delta_{\beta}$} & \multirow[b]{2}{*}{$\delta_{\mu}$} & \multicolumn{2}{|c|}{$\lambda^{\prime}=0.25$} & \multicolumn{2}{|c|}{$\lambda^{\prime}=0.50$} & \multicolumn{2}{|c|}{$\lambda^{\prime}=0.75$} \\
\hline & & $(7)$ & $(8)$ & $(7)$ & $(8)$ & $(7)$ & $(8)$ \\
\hline \multirow[t]{3}{*}{-} & 2.5 & 24.43 & 76.36 & 28.03 & 76.94 & 24.99 & 76.31 \\
\hline & 5.0 & 35.43 & 98.58 & 44.64 & 98.73 & 35.67 & 98.76 \\
\hline & 10.0 & 46.49 & 100.00 & 63.79 & 100.00 & 46.97 & 100.00 \\
\hline \multicolumn{8}{|c|}{ Panel B. Model $2 a$} \\
\hline \multirow[b]{2}{*}{$\delta_{\beta}$} & \multirow[b]{2}{*}{$\delta_{\mu}$} & \multicolumn{2}{|c|}{$\lambda^{\prime}=0.25$} & \multicolumn{2}{|c|}{$\lambda^{\prime}=0.50$} & \multicolumn{2}{|c|}{$\lambda^{\prime}=0.75$} \\
\hline & & $(7)$ & $(8)$ & $(7)$ & $(8)$ & $(7)$ & $(8)$ \\
\hline \multirow[t]{3}{*}{ - } & 2.5 & 31.80 & 76.29 & 20.93 & 76.85 & 32.16 & 76.17 \\
\hline & 5.0 & 49.57 & 98.54 & 36.30 & 98.72 & 50.64 & 98.72 \\
\hline & 10.0 & 68.99 & 100.00 & 57.21 & 100.00 & 70.21 & 100.00 \\
\hline \multicolumn{8}{|c|}{ Panel C. Model $2 b$} \\
\hline & & \multicolumn{2}{|c|}{$\lambda^{\prime}=0.25$} & \multicolumn{2}{|c|}{$\lambda^{\prime}=0.50$} & \multicolumn{2}{|c|}{$\lambda^{\prime}=0.75$} \\
\hline$\delta_{\beta}$ & $\delta_{\mu}$ & $(7)$ & $(8)$ & $(7)$ & $(8)$ & (7) & $(8)$ \\
\hline 1 & - & 53.91 & 71.75 & 70.78 & 79.28 & 56.18 & 72.21 \\
\hline 2 & - & 85.24 & 96.67 & 95.81 & 98.83 & 86.56 & 97.13 \\
\hline 4 & - & 98.98 & 100.00 & 99.99 & 100.00 & 99.39 & 100.00 \\
\hline \multicolumn{8}{|c|}{ Panel D. Model 2} \\
\hline \multirow[b]{2}{*}{$\delta_{\beta}$} & \multirow[b]{2}{*}{$\delta_{\mu}$} & \multicolumn{2}{|c|}{$\lambda^{\prime}=0.25$} & \multicolumn{2}{|c|}{$\lambda^{\prime}=0.50$} & \multicolumn{2}{|c|}{$\lambda^{\prime}=0.75$} \\
\hline & & $(7)$ & $(8)$ & $(7)$ & $(8)$ & $(7)$ & $(8)$ \\
\hline \multirow[t]{3}{*}{0} & 2.5 & 21.09 & 73.06 & 31.46 & 75.45 & 21.43 & 73.48 \\
\hline & 5.0 & 33.34 & 98.37 & 50.56 & 98.60 & 33.49 & 98.61 \\
\hline & 10.0 & 39.68 & 100.00 & 72.15 & 100.00 & 40.69 & 100.00 \\
\hline \multirow[t]{4}{*}{1} & 0.0 & 14.05 & 20.23 & 19.68 & 20.89 & 15.35 & 20.72 \\
\hline & 2.5 & 17.31 & 80.79 & 19.79 & 80.76 & 13.91 & 79.08 \\
\hline & 5.0 & 33.82 & 98.98 & 50.93 & 99.08 & 30.65 & 98.87 \\
\hline & 10.0 & 39.93 & 100.00 & 73.44 & 100.00 & 41.14 & 100.00 \\
\hline \multirow[t]{4}{*}{2} & 0.0 & 16.97 & 34.93 & 23.67 & 36.18 & 18.57 & 36.87 \\
\hline & 2.5 & 5.67 & 84.65 & 10.48 & 84.65 & 9.73 & 83.22 \\
\hline & 5.0 & 25.39 & 99.08 & 31.93 & 99.20 & 15.92 & 98.91 \\
\hline & 10.0 & 40.13 & 100.00 & 73.81 & 100.00 & 40.24 & 100.00 \\
\hline 4 & 0.0 & 19.88 & 47.11 & 28.38 & 48.47 & 21.45 & 49.18 \\
\hline & 2.5 & 6.93 & 87.72 & 10.70 & 86.94 & 8.77 & 85.99 \\
\hline & 5.0 & 9.07 & 99.12 & 10.22 & 99.18 & 4.67 & 99.07 \\
\hline & 10.0 & 33.83 & 100.00 & 40.95 & 100.00 & 12.91 & 100.00 \\
\hline
\end{tabular}


Table VI. Estimated powers of nominal 5\%-level Model 1 stationarity tests when a structural break occurs under the alternative: $T=100, \delta_{\mu}=\theta \sqrt{\left(\sigma_{\eta}^{2}+2\right) / 2}$

\begin{tabular}{|c|c|c|c|c|c|c|c|c|}
\hline$\sigma_{\eta}^{2}$ & $\theta$ & $\lambda^{\prime}$ & $\tilde{\xi}_{1}$ & $\tilde{\xi}_{1}^{*}$ & $\xi_{1}(\hat{\lambda})$ & $\xi_{1}(\hat{\lambda})^{*}$ & $s_{1}$ & $s_{1}^{*}$ \\
\hline \multirow[t]{10}{*}{0.01} & 0.0 & - & 29.96 & 6.74 & 18.90 & 16.04 & 12.02 & 9.32 \\
\hline & 2.5 & 0.25 & 63.74 & 29.70 & 54.04 & 45.43 & 37.23 & 32.37 \\
\hline & & 0.50 & 47.99 & 19.46 & 34.39 & 40.85 & 21.96 & 31.03 \\
\hline & & 0.75 & 62.89 & 28.99 & 53.08 & 45.40 & 37.02 & 33.13 \\
\hline & 5.0 & 0.25 & 61.67 & 24.33 & 54.77 & 46.21 & 50.39 & 43.98 \\
\hline & & 0.50 & 45.31 & 18.60 & 35.94 & 42.29 & 30.13 & 40.46 \\
\hline & & 0.75 & 60.67 & 23.97 & 53.80 & 45.97 & 50.88 & 45.48 \\
\hline & 10.0 & 0.25 & 54.75 & 15.30 & 54.81 & 46.28 & 51.92 & 45.56 \\
\hline & & 0.50 & 38.41 & 14.40 & 36.05 & 42.40 & 31.86 & 41.95 \\
\hline & & 0.75 & 53.98 & 15.59 & 53.88 & 45.99 & 51.66 & 46.33 \\
\hline \multirow[t]{10}{*}{0.10} & 0.0 & - & 87.67 & 59.76 & 73.51 & 70.25 & 44.75 & 46.02 \\
\hline & 2.5 & 0.25 & 93.65 & 71.33 & 85.59 & 81.07 & 57.39 & 57.73 \\
\hline & & 0.50 & 92.29 & 71.29 & 82.81 & 83.49 & 53.35 & 59.75 \\
\hline & & 0.75 & 93.66 & 71.62 & 85.60 & 81.82 & 56.17 & 57.60 \\
\hline & 5.0 & 0.25 & 94.33 & 73.12 & 91.25 & 86.83 & 73.16 & 72.93 \\
\hline & & 0.50 & 92.51 & 75.71 & 88.69 & 90.96 & 70.84 & 78.50 \\
\hline & & 0.75 & 94.78 & 73.75 & 91.78 & 88.12 & 72.83 & 73.21 \\
\hline & 10.0 & 0.25 & 91.65 & 62.26 & 91.51 & 87.15 & 88.83 & 86.21 \\
\hline & & 0.50 & 87.96 & 65.96 & 89.12 & 91.44 & 85.87 & 90.29 \\
\hline & & 0.75 & 91.99 & 62.14 & 92.08 & 88.40 & 88.99 & 86.81 \\
\hline \multirow[t]{10}{*}{1.00} & 0.0 & - & 99.55 & 92.73 & 97.10 & 95.90 & 77.81 & 83.20 \\
\hline & 2.5 & 0.25 & 99.50 & 92.86 & 97.37 & 96.04 & 78.67 & 83.91 \\
\hline & & 0.50 & 99.57 & 93.29 & 97.44 & 96.48 & 78.53 & 84.38 \\
\hline & & 0.75 & 99.67 & 92.76 & 97.28 & 95.84 & 79.25 & 84.61 \\
\hline & 5.0 & 0.25 & 99.71 & 93.35 & 98.17 & 96.92 & 81.53 & 86.84 \\
\hline & & 0.50 & 99.55 & 94.64 & 97.96 & 97.39 & 79.74 & 86.27 \\
\hline & & 0.75 & 99.68 & 92.97 & 98.20 & 96.84 & 80.58 & 86.45 \\
\hline & 10.0 & 0.25 & 99.58 & 90.79 & 99.24 & 98.47 & 87.60 & 91.68 \\
\hline & & 0.50 & 99.43 & 94.46 & 99.00 & 99.13 & 86.32 & 92.73 \\
\hline & & 0.75 & 99.56 & 90.95 & 99.24 & 98.29 & 87.76 & 91.73 \\
\hline
\end{tabular}


Table VII. Empirical sizes of nominal 5\%-level stationarity tests when a smooth transition occurs under the null: $T=100$; for Model 2: $\delta_{\mu}=\theta-\delta_{\beta} \lambda^{\prime} T$

\begin{tabular}{|c|c|c|c|c|c|c|c|c|c|}
\hline \multicolumn{10}{|c|}{ Panel A. Model $2 a$} \\
\hline$\delta_{\beta}$ & $\delta_{\mu}$ & $\gamma^{\prime}$ & $\lambda^{\prime}$ & $\tilde{\xi}_{2 a}$ & $\tilde{\xi}_{2 a}^{*}$ & $\xi_{2 a}(\hat{\lambda})$ & $\xi_{2 a}(\hat{\lambda})^{*}$ & $s_{2 a}$ & $s_{2 a}^{*}$ \\
\hline \multirow[t]{36}{*}{-} & \multirow[t]{12}{*}{2.5} & \multirow[t]{3}{*}{0.05} & 0.25 & 8.36 & 0.08 & 5.31 & 1.66 & 3.75 & 0.81 \\
\hline & & & 0.50 & 6.77 & 0.03 & 5.99 & 2.08 & 5.76 & 1.11 \\
\hline & & & 0.75 & 8.32 & 0.09 & 5.60 & 1.79 & 3.42 & 0.61 \\
\hline & & \multirow[t]{3}{*}{0.10} & 0.25 & 34.69 & 3.48 & 15.05 & 8.01 & 2.25 & 0.75 \\
\hline & & & 0.50 & 22.27 & 0.36 & 15.21 & 5.72 & 10.30 & 2.70 \\
\hline & & & 0.75 & 35.13 & 3.82 & 15.37 & 7.95 & 2.09 & 0.50 \\
\hline & & \multirow[t]{3}{*}{0.50} & 0.25 & 38.50 & 5.33 & 14.37 & 8.51 & 3.88 & 1.26 \\
\hline & & & 0.50 & 32.94 & 0.77 & 20.03 & 6.20 & 11.35 & 2.44 \\
\hline & & & 0.75 & 39.25 & 5.55 & 15.22 & 8.90 & 3.93 & 1.57 \\
\hline & & \multirow[t]{3}{*}{1.00} & 0.25 & 28.54 & 2.98 & 9.66 & 5.49 & 5.89 & 2.26 \\
\hline & & & 0.50 & 27.74 & 0.63 & 17.39 & 5.12 & 14.28 & 2.86 \\
\hline & & & 0.75 & 28.77 & 2.70 & 10.38 & 5.54 & 5.73 & 2.09 \\
\hline & \multirow[t]{12}{*}{5.0} & \multirow[t]{3}{*}{0.05} & 0.25 & 28.06 & 2.06 & 11.69 & 5.38 & 3.46 & 1.34 \\
\hline & & & 0.50 & 12.90 & 0.15 & 9.75 & 3.68 & 8.90 & 1.85 \\
\hline & & & 0.75 & 27.81 & 1.92 & 12.05 & 5.62 & 2.77 & 0.86 \\
\hline & & \multirow[t]{3}{*}{0.10} & 0.25 & 96.56 & 71.69 & 83.98 & 75.06 & 1.35 & 0.71 \\
\hline & & & 0.50 & 69.29 & 4.52 & 44.42 & 18.92 & 12.16 & 7.36 \\
\hline & & & 0.75 & 96.79 & 73.08 & 85.54 & 76.64 & 0.97 & 0.45 \\
\hline & & \multirow[t]{3}{*}{0.50} & 0.25 & 80.77 & 32.23 & 50.55 & 38.11 & 5.19 & 2.08 \\
\hline & & & 0.50 & 67.60 & 2.32 & 39.21 & 13.28 & 11.64 & 2.13 \\
\hline & & & 0.75 & 81.94 & 32.24 & 50.93 & 38.51 & 5.24 & 2.40 \\
\hline & & \multirow[t]{3}{*}{1.00} & 0.25 & 48.66 & 8.66 & 19.49 & 11.79 & 8.09 & 3.28 \\
\hline & & & 0.50 & 39.52 & 0.75 & 22.88 & 6.92 & 15.62 & 3.58 \\
\hline & & & 0.75 & 49.37 & 9.02 & 20.46 & 12.79 & 8.13 & 3.27 \\
\hline & \multirow[t]{12}{*}{10.0} & \multirow[t]{3}{*}{0.05} & 0.25 & 92.96 & 55.73 & 73.17 & 60.99 & 1.81 & 1.16 \\
\hline & & & 0.50 & 39.98 & 1.07 & 24.92 & 10.52 & 20.16 & 8.83 \\
\hline & & & 0.75 & 92.39 & 54.10 & 72.17 & 59.49 & 2.20 & 1.25 \\
\hline & & \multirow[t]{3}{*}{0.10} & 0.25 & 100.00 & 100.00 & 100.00 & 100.00 & 0.16 & 0.08 \\
\hline & & & 0.50 & 99.92 & 74.69 & 98.32 & 82.61 & 1.68 & 0.72 \\
\hline & & & 0.75 & 100.00 & 100.00 & 100.00 & 100.00 & 0.07 & 0.02 \\
\hline & & \multirow[t]{3}{*}{0.50} & 0.25 & 99.97 & 95.99 & 98.82 & 96.63 & 4.97 & 2.25 \\
\hline & & & 0.50 & 99.70 & 30.90 & 91.36 & 49.84 & 11.74 & 2.12 \\
\hline & & & 0.75 & 99.97 & 96.63 & 98.96 & 97.32 & 4.82 & 2.34 \\
\hline & & \multirow[t]{3}{*}{1.00} & 0.25 & 88.29 & 37.14 & 57.61 & 43.17 & 8.09 & 3.29 \\
\hline & & & 0.50 & 77.80 & 1.72 & 42.94 & 11.75 & 16.06 & 3.48 \\
\hline & & & 0.75 & 89.21 & 37.31 & 58.07 & 43.89 & 7.84 & 3.32 \\
\hline \multicolumn{10}{|c|}{ Panel B. Model 2} \\
\hline$\delta_{\beta}$ & $\theta$ & $\gamma^{\prime}$ & $\lambda^{\prime}$ & $\tilde{\xi}_{2}$ & $\tilde{\xi}_{2}^{*}$ & $\xi_{2}(\hat{\lambda})$ & $\xi_{2}(\hat{\lambda})^{*}$ & $s_{2}$ & $s_{2}^{*}$ \\
\hline \multirow[t]{3}{*}{1} & \multirow[t]{3}{*}{0.0} & \multirow[t]{3}{*}{0.05} & 0.25 & 100.00 & 99.97 & 100.00 & 99.97 & 1.65 & 1.69 \\
\hline & & & 0.50 & 100.00 & 100.00 & 100.00 & 100.00 & 0.30 & 0.32 \\
\hline & & & 0.75 & 100.00 & 99.99 & 100.00 & 99.99 & 0.64 & 0.67 \\
\hline
\end{tabular}




\begin{tabular}{|c|c|c|c|c|c|c|c|c|c|}
\hline \multicolumn{10}{|c|}{ Panel B. Model 2 contd. } \\
\hline$\delta_{\beta}$ & $\theta$ & $\gamma^{\prime}$ & $\lambda^{\prime}$ & $\tilde{\xi}_{2}$ & $\tilde{\xi}_{2}^{*}$ & $\xi_{2}(\hat{\lambda})$ & $\xi_{2}(\hat{\lambda})^{*}$ & $s_{2}$ & $s_{2}^{*}$ \\
\hline \multirow[t]{45}{*}{1} & 0.0 & 0.10 & 0.25 & 98.59 & 51.15 & 89.63 & 58.58 & 0.25 & 0.16 \\
\hline & & & 0.50 & 100.00 & 99.39 & 99.79 & 99.59 & 0.01 & 0.15 \\
\hline & & & 0.75 & 98.66 & 51.46 & 90.91 & 59.05 & 0.28 & 0.20 \\
\hline & & 0.50 & 0.25 & 34.67 & 1.57 & 21.06 & 5.74 & 8.55 & 2.76 \\
\hline & & & 0.50 & 18.97 & 1.51 & 7.34 & 5.10 & 1.46 & 2.05 \\
\hline & & & 0.75 & 33.27 & 1.33 & 19.58 & 5.44 & 8.21 & 2.64 \\
\hline & & 1.00 & 0.25 & 33.16 & 1.77 & 18.81 & 4.67 & 9.82 & 3.28 \\
\hline & & & 0.50 & 17.24 & 1.44 & 6.27 & 4.26 & 2.59 & 3.03 \\
\hline & & & 0.75 & 31.88 & 1.73 & 18.19 & 4.38 & 8.97 & 2.87 \\
\hline & 2.5 & 0.05 & 0.25 & 100.00 & 99.90 & 99.99 & 99.95 & 1.65 & 1.65 \\
\hline & & & 0.50 & 100.00 & 100.00 & 100.00 & 100.00 & 0.27 & 0.29 \\
\hline & & & 0.75 & 100.00 & 100.00 & 100.00 & 100.00 & 0.77 & 0.81 \\
\hline & & 0.10 & 0.25 & 99.26 & 68.08 & 94.98 & 73.86 & 0.25 & 0.18 \\
\hline & & & 0.50 & 99.99 & 99.25 & 99.68 & 99.39 & 0.01 & 0.14 \\
\hline & & & 0.75 & 99.11 & 49.63 & 91.10 & 58.56 & 0.28 & 0.22 \\
\hline & & 0.50 & 0.25 & 52.33 & 5.71 & 38.28 & 14.11 & 10.37 & 3.48 \\
\hline & & & 0.50 & 28.43 & 2.96 & 16.07 & 12.10 & 1.70 & 2.21 \\
\hline & & & 0.75 & 31.70 & 1.70 & 17.79 & 4.73 & 9.29 & 3.32 \\
\hline & & 1.00 & 0.25 & 37.25 & 2.63 & 22.56 & 5.58 & 11.33 & 3.49 \\
\hline & & & 0.50 & 17.81 & 1.20 & 7.85 & 5.53 & 2.21 & 2.69 \\
\hline & & & 0.75 & 30.26 & 1.76 & 18.07 & 4.61 & 9.87 & 3.23 \\
\hline & 5.0 & 0.05 & 0.25 & 100.00 & 99.74 & 99.95 & 99.83 & 1.47 & 1.44 \\
\hline & & & 0.50 & 100.00 & 100.00 & 100.00 & 100.00 & 0.18 & 0.26 \\
\hline & & & 0.75 & 100.00 & 100.00 & 100.00 & 100.00 & 0.61 & 0.60 \\
\hline & & 0.10 & 0.25 & 99.81 & 87.75 & 98.87 & 90.99 & 0.17 & 0.16 \\
\hline & & & 0.50 & 99.96 & 99.17 & 99.63 & 99.28 & 0.00 & 0.13 \\
\hline & & & 0.75 & 99.69 & 60.10 & 94.32 & 69.66 & 0.31 & 0.20 \\
\hline & & 0.50 & 0.25 & 81.98 & 14.98 & 68.52 & 39.90 & 8.65 & 3.18 \\
\hline & & & 0.50 & 70.73 & 19.94 & 51.43 & 44.11 & 1.24 & 2.08 \\
\hline & & & 0.75 & 39.76 & 2.00 & 23.67 & 6.22 & 10.85 & 3.95 \\
\hline & & 1.00 & 0.25 & 49.94 & 3.64 & 33.29 & 10.51 & 12.22 & 3.90 \\
\hline & & & 0.50 & 36.21 & 4.88 & 17.57 & 13.38 & 2.51 & 3.19 \\
\hline & & & 0.75 & 34.73 & 1.64 & 22.33 & 5.77 & 12.08 & 4.20 \\
\hline & 10.0 & 0.05 & 0.25 & 100.00 & 97.42 & 99.82 & 98.44 & 1.05 & 1.07 \\
\hline & & & 0.50 & 100.00 & 100.00 & 100.00 & 100.00 & 0.23 & 0.25 \\
\hline & & & 0.75 & 100.00 & 100.00 & 100.00 & 100.00 & 0.61 & 0.64 \\
\hline & & 0.10 & 0.25 & 100.00 & 99.90 & 100.00 & 99.99 & 0.13 & 0.14 \\
\hline & & & 0.50 & 99.99 & 99.18 & 99.63 & 99.36 & 0.00 & 0.13 \\
\hline & & & 0.75 & 99.97 & 89.44 & 99.03 & 92.66 & 0.31 & 0.16 \\
\hline & & 0.50 & 0.25 & 99.74 & 52.48 & 96.92 & 80.66 & 8.05 & 2.70 \\
\hline & & & 0.50 & 99.97 & 97.77 & 99.08 & 98.40 & 1.24 & 1.99 \\
\hline & & & 0.75 & 96.40 & 13.15 & 76.05 & 30.46 & 9.93 & 3.66 \\
\hline & & 1.00 & 0.25 & 79.54 & 5.73 & 57.99 & 25.83 & 11.87 & 4.11 \\
\hline & & & 0.50 & 86.62 & 40.06 & 55.34 & 47.61 & 2.43 & 3.28 \\
\hline & & & 0.75 & 76.27 & 5.63 & 45.27 & 14.98 & 12.39 & 4.21 \\
\hline
\end{tabular}




\begin{tabular}{|c|c|c|c|c|c|c|c|c|c|}
\hline \multicolumn{10}{|c|}{ Panel B. Model 2 contd. } \\
\hline$\delta_{\beta}$ & $\theta$ & $\gamma^{\prime}$ & $\lambda^{\prime}$ & $\tilde{\xi}_{2}$ & $\tilde{\xi}_{2}^{*}$ & $\xi_{2}(\hat{\lambda})$ & $\xi_{2}(\hat{\lambda})^{*}$ & $s_{2}$ & $s_{2}^{*}$ \\
\hline \multirow[t]{48}{*}{2} & 0.0 & 0.05 & 0.25 & 100.00 & 100.00 & 100.00 & 100.00 & 0.07 & 0.12 \\
\hline & & & 0.50 & 100.00 & 100.00 & 100.00 & 100.00 & 0.04 & 0.09 \\
\hline & & & 0.75 & 100.00 & 100.00 & 100.00 & 100.00 & 0.05 & 0.08 \\
\hline & & 0.10 & 0.25 & 100.00 & 99.98 & 100.00 & 100.00 & 0.11 & 0.11 \\
\hline & & & 0.50 & 100.00 & 100.00 & 100.00 & 100.00 & 0.00 & 0.14 \\
\hline & & & 0.75 & 100.00 & 100.00 & 100.00 & 100.00 & 0.13 & 0.11 \\
\hline & & 0.50 & 0.25 & 41.64 & 1.16 & 36.26 & 13.43 & 9.34 & 3.02 \\
\hline & & & 0.50 & 30.46 & 2.48 & 20.95 & 16.61 & 1.10 & 1.78 \\
\hline & & & 0.75 & 40.19 & 1.08 & 34.83 & 13.01 & 9.09 & 3.01 \\
\hline & & 1.00 & 0.25 & 31.90 & 1.36 & 19.38 & 4.79 & 12.02 & 3.95 \\
\hline & & & 0.50 & 16.02 & 1.05 & 6.51 & 4.49 & 2.14 & 2.77 \\
\hline & & & 0.75 & 30.66 & 1.23 & 18.58 & 4.80 & 11.45 & 3.55 \\
\hline & 2.5 & 0.05 & 0.25 & 100.00 & 100.00 & 100.00 & 100.00 & 0.10 & 0.10 \\
\hline & & & 0.50 & 100.00 & 100.00 & 100.00 & 100.00 & 0.02 & 0.11 \\
\hline & & & 0.75 & 100.00 & 100.00 & 100.00 & 100.00 & 0.00 & 0.04 \\
\hline & & 0.10 & 0.25 & 100.00 & 99.99 & 100.00 & 100.00 & 0.11 & 0.11 \\
\hline & & & 0.50 & 100.00 & 100.00 & 100.00 & 100.00 & 0.00 & 0.13 \\
\hline & & & 0.75 & 100.00 & 99.99 & 100.00 & 100.00 & 0.13 & 0.11 \\
\hline & & 0.50 & 0.25 & 58.90 & 2.58 & 62.36 & 32.35 & 9.20 & 3.14 \\
\hline & & & 0.50 & 38.60 & 3.64 & 32.69 & 26.61 & 1.34 & 1.99 \\
\hline & & & 0.75 & 35.18 & 1.09 & 23.53 & 7.16 & 9.13 & 3.30 \\
\hline & & 1.00 & 0.25 & 33.17 & 1.00 & 24.82 & 6.85 & 11.90 & 4.01 \\
\hline & & & 0.50 & 16.46 & 0.97 & 8.60 & 6.43 & 2.62 & 3.27 \\
\hline & & & 0.75 & 30.16 & 1.43 & 18.21 & 4.70 & 12.19 & 4.13 \\
\hline & 5.0 & 0.05 & 0.25 & 100.00 & 100.00 & 100.00 & 100.00 & 0.13 & 0.18 \\
\hline & & & 0.50 & 100.00 & 100.00 & 100.00 & 100.00 & 0.02 & 0.05 \\
\hline & & & 0.75 & 100.00 & 100.00 & 100.00 & 100.00 & 0.05 & 0.10 \\
\hline & & 0.10 & 0.25 & 100.00 & 100.00 & 100.00 & 100.00 & 0.11 & 0.11 \\
\hline & & & 0.50 & 100.00 & 100.00 & 100.00 & 100.00 & 0.00 & 0.13 \\
\hline & & & 0.75 & 100.00 & 99.99 & 100.00 & 99.99 & 0.14 & 0.11 \\
\hline & & 0.50 & 0.25 & 88.29 & 22.47 & 88.57 & 64.59 & 8.14 & 2.90 \\
\hline & & & 0.50 & 66.25 & 11.27 & 65.73 & 58.73 & 1.60 & 2.45 \\
\hline & & & 0.75 & 38.71 & 1.26 & 23.47 & 6.02 & 10.45 & 3.93 \\
\hline & & 1.00 & 0.25 & 46.83 & 2.78 & 39.33 & 14.03 & 11.47 & 3.67 \\
\hline & & & 0.50 & 24.03 & 1.13 & 18.51 & 14.32 & 2.55 & 3.25 \\
\hline & & & 0.75 & 30.94 & 1.22 & 20.95 & 5.17 & 13.13 & 4.40 \\
\hline & 10.0 & 0.05 & 0.25 & 100.00 & 100.00 & 100.00 & 100.00 & 0.13 & 0.22 \\
\hline & & & 0.50 & 100.00 & 100.00 & 100.00 & 100.00 & 0.01 & 0.04 \\
\hline & & & 0.75 & 100.00 & 100.00 & 100.00 & 100.00 & 0.06 & 0.06 \\
\hline & & 0.10 & 0.25 & 100.00 & 100.00 & 100.00 & 100.00 & 0.11 & 0.11 \\
\hline & & & 0.50 & 100.00 & 100.00 & 100.00 & 100.00 & 0.00 & 0.12 \\
\hline & & & 0.75 & 100.00 & 100.00 & 100.00 & 100.00 & 0.15 & 0.13 \\
\hline & & 0.50 & 0.25 & 99.98 & 69.33 & 99.52 & 94.90 & 7.67 & 2.58 \\
\hline & & & 0.50 & 99.97 & 90.17 & 99.64 & 99.32 & 1.48 & 2.34 \\
\hline & & & 0.75 & 77.66 & 2.39 & 58.48 & 14.87 & 11.46 & 4.53 \\
\hline & & 1.00 & 0.25 & 82.38 & 5.67 & 64.22 & 32.47 & 12.16 & 3.91 \\
\hline & & & 0.50 & 86.32 & 29.24 & 57.57 & 49.72 & 2.42 & 3.28 \\
\hline & & & 0.75 & 63.80 & 1.21 & 40.87 & 11.61 & 12.53 & 4.39 \\
\hline
\end{tabular}


Table VIII. Empirical applications of stationarity tests

\begin{tabular}{|c|c|c|c|c|}
\hline \multicolumn{5}{|c|}{ Panel A. $S \& P 500$ index } \\
\hline & $\tilde{\xi}_{i}$ & $\xi_{i}(\hat{\lambda})$ & $\xi_{i}(\hat{\lambda})^{*}$ & $s_{i}^{*}$ \\
\hline Model 1 & $\begin{array}{c}0.534^{* * *} \\
(\hat{\tau}=1948)\end{array}$ & \multicolumn{2}{|c|}{$(\hat{\tau}=1954)$} & $(\hat{\lambda}=7.464, \hat{\gamma}=0.020)$ \\
\hline Model $2 a$ & $\begin{array}{c}0.228^{* * *} \\
(\hat{\tau}=1966)\end{array}$ & \multicolumn{2}{|c|}{$(\hat{\tau}=1912)$} & $(\hat{\lambda}=4.533, \hat{\gamma}=0.020)$ \\
\hline Model $2 b$ & $\begin{array}{l}0.057 \\
(\hat{\tau}=1945)\end{array}$ & \multicolumn{2}{|c|}{$\begin{array}{c}0.059 \quad 0.059 \\
(\hat{\tau}=1943)\end{array}$} & \\
\hline Model 2 & $\begin{array}{c}0.056^{* * *} \\
(\hat{\tau}=1935)\end{array}$ & \multicolumn{2}{|c|}{$(\hat{\tau}=1939)$} & $(\hat{\lambda}=0.617, \hat{\gamma}=1.175)$ \\
\hline \multicolumn{5}{|c|}{ Panel B. UK GDP } \\
\hline & $\tilde{\xi}_{i}$ & $\xi_{i}(\hat{\lambda})$ & $\xi_{i}(\hat{\lambda})^{*}$ & $s_{i}^{*}$ \\
\hline Model 1 & $\begin{array}{c}0.779^{* * *} \\
(\hat{\tau}=1928)\end{array}$ & \multicolumn{2}{|c|}{$(\hat{\tau}=1939)$} & $(\hat{\lambda}=33.37, \hat{\gamma}=0.004)$ \\
\hline Model $2 a$ & $\begin{array}{c}0.137^{* * *} \\
(\hat{\tau}=1912)\end{array}$ & $\begin{array}{r}0.282^{* * *} \\
(\hat{\tau}=\end{array}$ & $\begin{array}{l}0.282^{* * *} \\
1918)\end{array}$ & $\begin{array}{cc}0.293^{* * *} & 0.293^{* * *} \\
(\hat{\lambda}=0.448, \hat{\gamma}=1.815)\end{array}$ \\
\hline Model $2 b$ & $\begin{array}{c}0.147^{* * *} \\
(\hat{\tau}=1958)\end{array}$ & \multicolumn{2}{|c|}{$\begin{array}{c}0.157^{* * *} \quad 0.157^{* * *} \\
(\hat{\tau}=1950)\end{array}$} & \\
\hline Model 2 & $\begin{array}{l}0.036 \\
(\hat{\tau}=1919)\end{array}$ & \multicolumn{2}{|c|}{$(\hat{\tau}=1919)$} & $(\hat{\lambda}=0.451, \hat{\gamma}=1.978)$ \\
\hline \multicolumn{5}{|c|}{ Panel C. British industrial production } \\
\hline & $\tilde{\xi}_{i}$ & $\xi_{i}(\hat{\lambda})$ & $\xi_{i}(\hat{\lambda})^{*}$ & $s_{i}^{*}$ \\
\hline Model 1 & $\begin{array}{c}0.990^{* * *} \\
(\hat{\tau}=1820)\end{array}$ & $\begin{array}{r}1.155^{* * *} \\
(\hat{\tau}=\end{array}$ & $\begin{array}{l}1.155^{* * *} \\
1832)\end{array}$ & $\begin{array}{cc}0.160 & 0.160^{*} \\
(\hat{\lambda}=0.679, \hat{\gamma}=0.031)\end{array}$ \\
\hline Model $2 a$ & $\begin{array}{c}0.434^{* * *} \\
(\hat{\tau}=1752)\end{array}$ & \multicolumn{2}{|c|}{$(\hat{\tau}=1744)$} & $(\hat{\lambda}=0.678, \hat{\gamma}=0.041)$ \\
\hline Model $2 b$ & $\begin{array}{c}0.195^{* * *} \\
(\hat{\tau}=1787)\end{array}$ & \multicolumn{2}{|c|}{$\begin{array}{c}0.210^{* * *} 0.210^{* * *} \\
(\hat{\tau}=1791)\end{array}$} & \\
\hline Model 2 & $\begin{array}{c}0.190^{* * *} \\
(\hat{\tau}=1780)\end{array}$ & $\begin{array}{r}0.215^{* * *} \\
(\hat{\tau}= \\
\end{array}$ & $(\hat{\tau}=1791)$ & $\begin{array}{cc}0.039 & 0.039 \\
(\hat{\lambda}=0.591, \hat{\gamma}=0.062)\end{array}$ \\
\hline \multicolumn{5}{|c|}{ Panel D. Dollar-sterling exchange rate } \\
\hline & $\tilde{\xi}_{i}$ & $\xi_{i}(\hat{\lambda})$ & $\xi_{i}(\hat{\lambda})^{*}$ & $s_{i}^{*}$ \\
\hline Model 1 & $\begin{array}{c}0.137^{* *} \\
(\hat{\tau}=1993: 5)\end{array}$ & \multicolumn{2}{|c|}{$(\hat{\tau}=1992: 9)$} & $(\hat{\lambda}=0.345, \hat{\gamma}=24.32)$ \\
\hline Model $2 a$ & $\begin{array}{c}0.088^{* * *} \\
(\hat{\tau}=1996: 5)\end{array}$ & $\begin{array}{l}0.182^{* * *} \\
\quad(\hat{\tau}=1\end{array}$ & $\begin{array}{l}0.182^{* * *} \\
92: 10)\end{array}$ & $\begin{array}{c}0.100^{* *} \quad 0.100^{*} \\
(\hat{\lambda}=0.625, \hat{\gamma}=0.309)\end{array}$ \\
\hline Model $2 b$ & $\begin{array}{c}0.122^{* * *} \\
(\hat{\tau}=1993: 8)\end{array}$ & $\begin{array}{l}0.123^{* * *} \\
\quad(\hat{\tau}=1\end{array}$ & $\begin{array}{l}0.123^{* * *} \\
994: 2)\end{array}$ & \\
\hline Model 2 & $\begin{array}{c}0.081^{* * *} \\
(\hat{\tau}=1996: 11)\end{array}$ & $\begin{array}{l}0.167^{* * *} \\
\quad(\hat{\tau}=1\end{array}$ & $\begin{array}{l}0.167^{* * *} \\
992: 9)\end{array}$ & $\begin{array}{c}0.082^{* *} \quad 0.082^{* *} \\
(\hat{\lambda}=0.680, \hat{\gamma}=0.142)\end{array}$ \\
\hline
\end{tabular}

Note: ${ }^{*},{ }^{* *}$ and ${ }^{* * *}$ denote significance at the $10 \%-, 5 \%-$ and $1 \%$-levels respectively. 

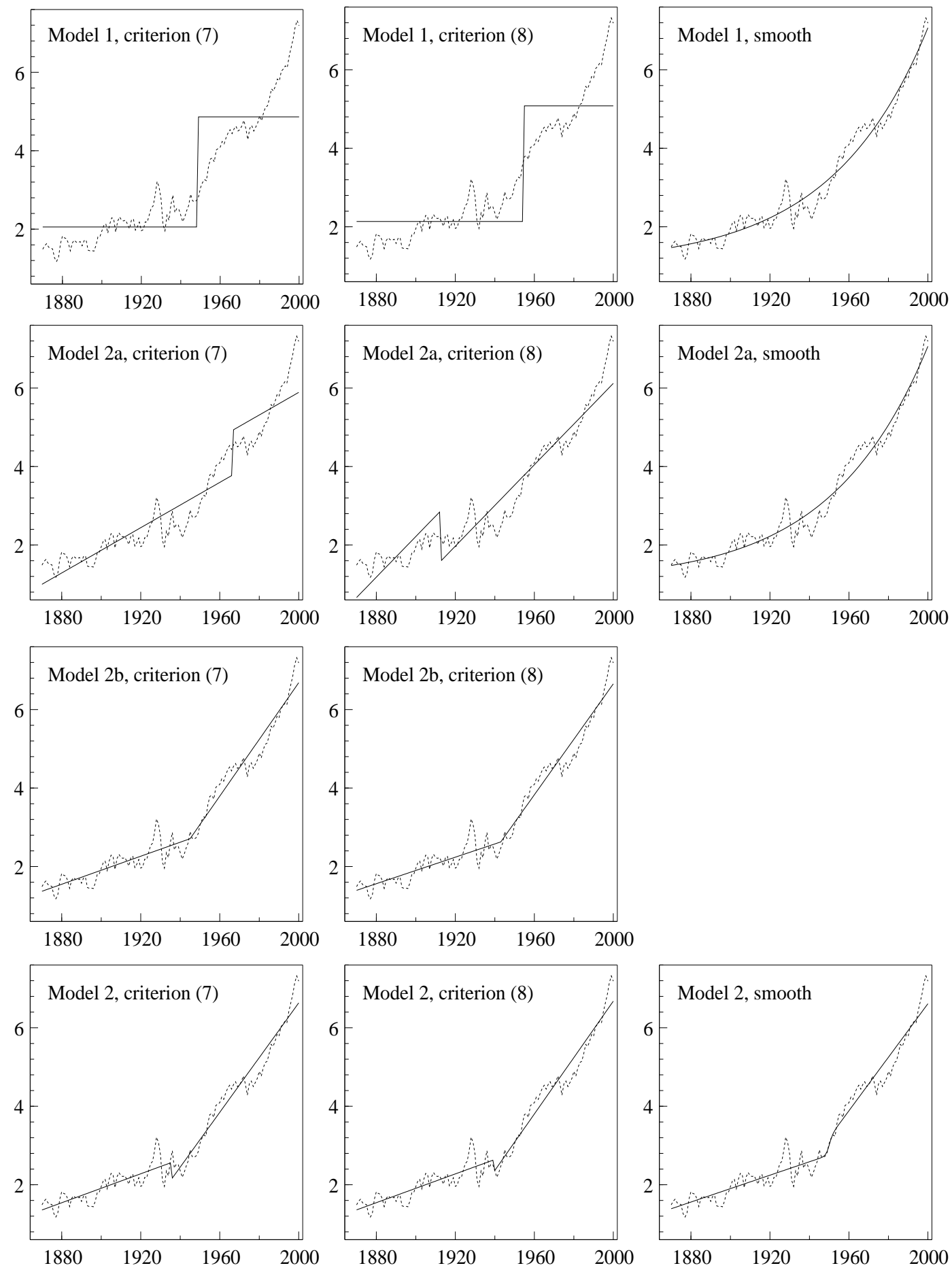

Figure 1. $S \& P P 500$ index and fitted trend functions 

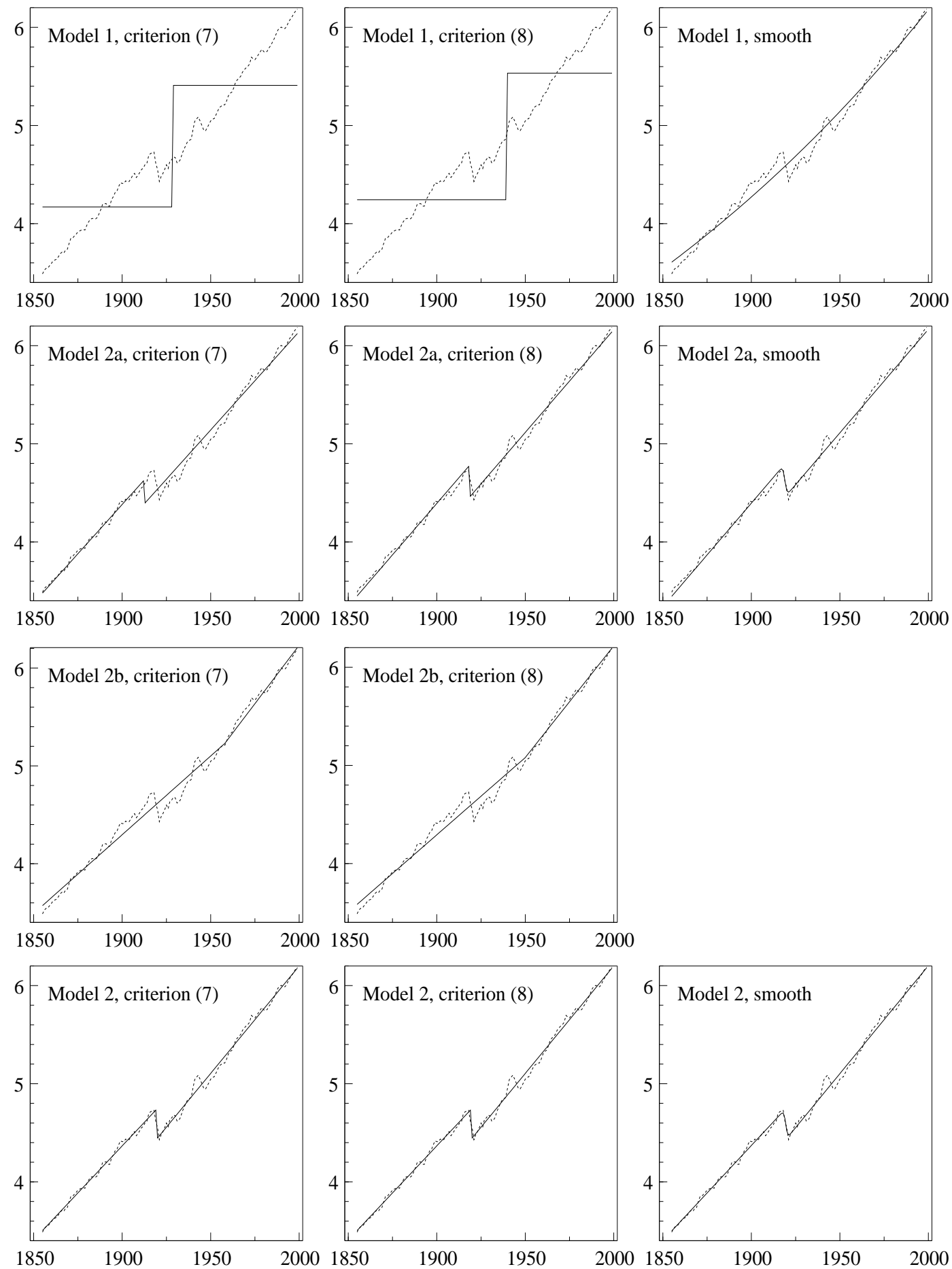

Figure 2. UK GDP and fitted trend functions 

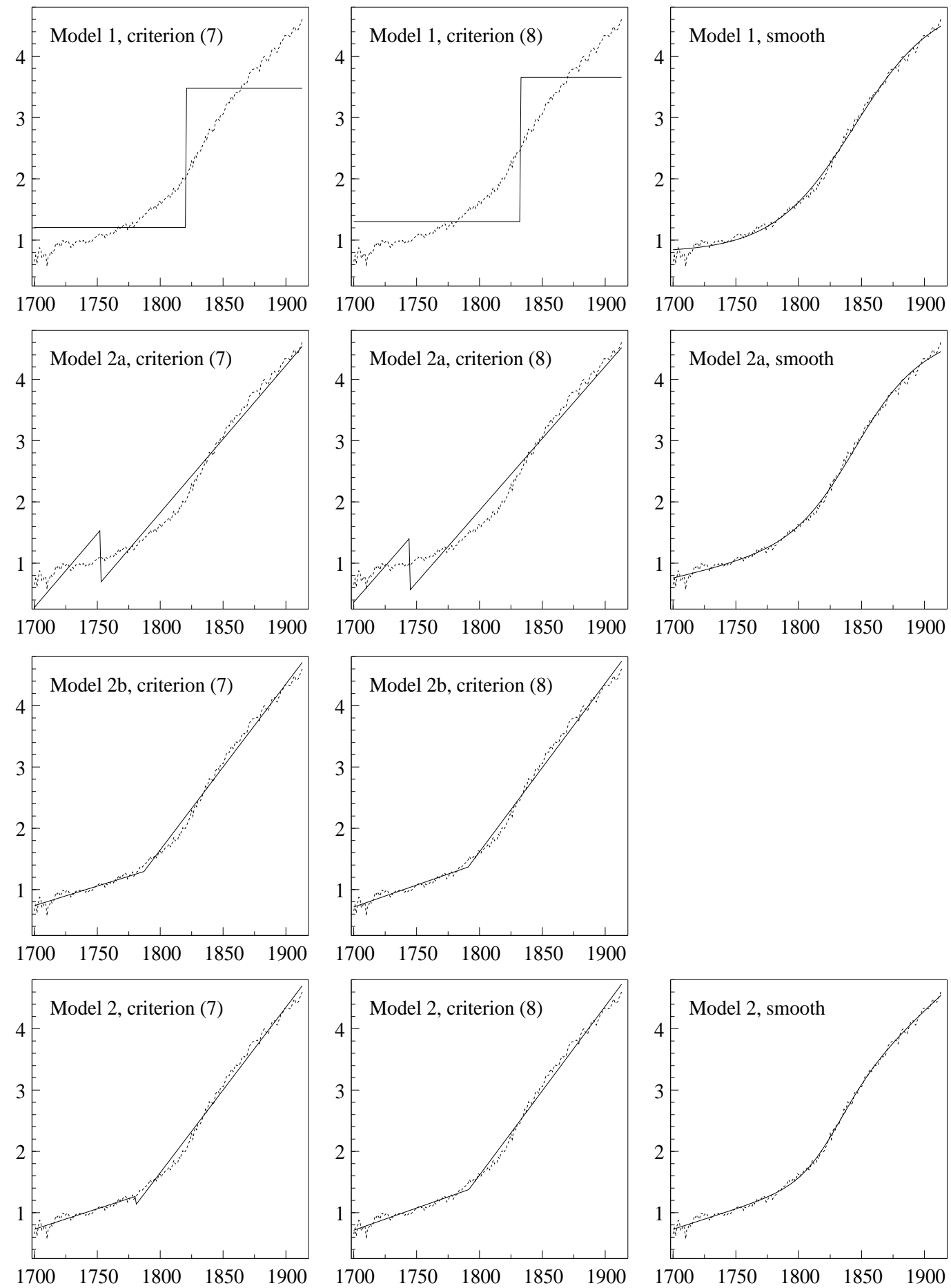

Figure 3. British industrial production and fitted trend functions 

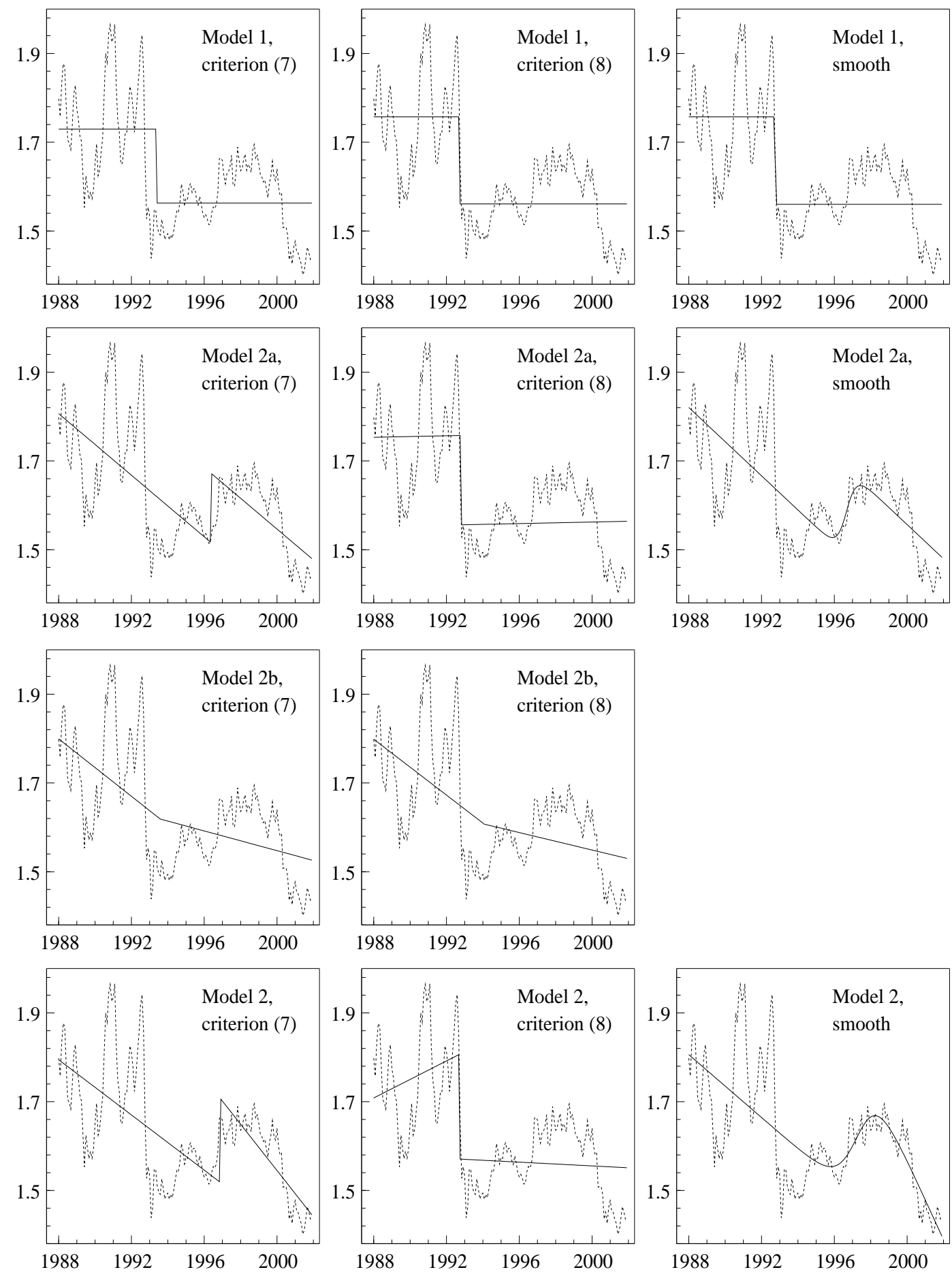

Figure 4. Dollar-sterling exchange rate and fitted trend functions 